\title{
A STOCHASTIC EVOLUTION EQUATION ARISING FROM THE FLUCTUATIONS OF A CLASS OF INTERACTING PARTICLE SYSTEMS *
}

\author{
THOMAS G. KURTZ † AND JIE XIONG $\ddagger$
}

Abstract. In an earlier paper, we studied the approximation of solutions $V(t)$ to a class of SPDEs by the empirical measure $V^{n}(t)$ of a system of $n$ interacting diffusions. In the present paper, we consider a central limit type problem, showing that $\sqrt{n}\left(V^{n}-V\right)$ converges weakly, in the dual of a nuclear space, to the unique solution of a stochastic evolution equation. Analogous results in which the diffusions that determine $V^{n}$ are replaced by their Euler approximations are also discussed.

Key words. Stochastic partial differential equations, interacting infinite particle system, central limit theorem, Euler scheme

MSC 2000 subject classifications: Primary 60H15, 60H35; Secondary 60B12, 60F17, 60F25, $60 \mathrm{H} 10,93 \mathrm{E} 11$.

\section{Introduction}

In [26], we considered a class of nonlinear stochastic partial differential equations (SPDE) of the form

$$
\begin{aligned}
d v(t, x)= & \left(\frac{1}{2} \sum_{i, j=1}^{d} \partial_{x_{i}} \partial_{x_{j}}\left[a_{i j}(x, v(t, \cdot)) v(t, x)\right]-\sum_{i=1}^{d} \partial_{x_{i}}\left[b_{i}(x, v(t, \cdot)) v(t, x)\right]\right. \\
& +d(x, v(t, \cdot)) v(t, x)) d t \\
& -\int_{U}\left(\beta(x, v(t, \cdot), u) v(t, x)+\sum_{i=1}^{d} \partial_{x_{i}}\left[\alpha_{i}(x, v(t, \cdot), u)\right]\right) W(d u d t) .
\end{aligned}
$$

The natural interpretation of $v$ is as the density of a mass distribution $V$ evolving in time, and in fact, since $v$ will not have the regularity presumed in (1.1), to rigorously formulate the equation, we must use a weak form

$$
\begin{aligned}
& \langle\phi, V(t)\rangle-\langle\phi, V(0)\rangle \\
& \quad=\int_{0}^{t}\langle\phi d(\cdot, V(s))+L(V(s)) \phi, V(s)\rangle d s \\
& \quad+\int_{U \times[0, t]}\left\langle\phi \beta(\cdot, V(s), u)+\nabla \phi^{T} \alpha(\cdot, V(s), u), V(s)\right\rangle W(d u d s) .
\end{aligned}
$$

where

$$
L(v) \phi(x)=\frac{1}{2} \sum_{i, j} a_{i j}(x, v) \partial_{x_{i}} \partial_{x_{j}} \phi(x)+\sum_{i} b_{i}(x, v) \partial_{x_{i}} \phi(x)
$$

${ }^{*}$ Received: February 4, 2003; accepted (in revised version): July 06, 2004. Communicated by Shi Jin.

${ }^{\dagger}$ Departments of Mathematics and Statistics, University of Wisconsin-Madison, 480 Lincoln Drive, Madison, WI 53706-1388, USA (kurtz@math.wisc.edu). Research supported in part by NSF grant DMS 02-05034.

$¥$ Department of Mathematics, University of Tennessee, Knoxville, TN 37996-1300, USA, and Department of Mathematics, Hebei Normal University, Shijiazhuang 050016, PRC (jxiong@math.utk.edu). Research supported partially by NSA and by Alexander von Humboldt Foundation. 
Equations in this class arise in a variety of settings, including nonlinear filtering with both the Zakai and the Kushner-FKK equations being of this form. Other examples include McKean-Vlasov equations [30] and classes of SPDEs considered by Kotelenez [23] and Dawson and Vaillancourt [9].

In [26], we established a representation of the solution of (1.1) in terms of weighted empirical measures of the form

$$
V(t)=\lim _{n \rightarrow \infty} \frac{1}{n} \sum_{i=1}^{n} A_{i}(t) \delta_{X_{i}(t)},
$$

where $\delta_{x}$ is the Dirac measure at $x$ and the limit exists in the weak ${ }^{*}$ topology on $\mathcal{M}\left(\mathbb{R}^{d}\right)$. To be precise, let $U$ be a Polish space and $\mu$ be a $\sigma$-finite measure on $U$. $W$ will be a space-time Gaussian white noise on $U \times[0, \infty)$ with covariance measure $\mu(d u) d t$, namely

$$
E(W(A \times[0, t]) W(B \times[0, s]))=\mu(A \cap B)(t \wedge s) .
$$

For $1 \leq i, j \leq d, a_{i j}, b_{i}, d$ and $\alpha_{i}, \beta$ will be real functions on $\mathbb{R}^{d} \times \mathcal{M}\left(\mathbb{R}^{d}\right)$ and on $\mathbb{R}^{d} \times \mathcal{M}\left(\mathbb{R}^{d}\right) \times U$ respectively. Here $\mathcal{M}\left(\mathbb{R}^{d}\right)$ is the collection of all finite measures on $\mathbb{R}^{d}$. Dot notation will represent a function in that variable alone. For example, $d(\cdot, v)$ is the real-valued function on $\mathbb{R}^{d}$ with $v \in \mathcal{M}\left(\mathbb{R}^{d}\right)$ fixed. $b$ and $\alpha$ will denote the vectors with components $b_{i}$ and $\alpha_{i}$ and $a$ will denote the matrix $\left(\left(a_{i j}\right)\right)$.

The particle system $\left\{X_{i}, A_{i}, V\right\}$ giving the solution is governed by the following equations:

$$
\begin{aligned}
X_{i}(t)= & X_{i}(0)+\int_{0}^{t} \sigma\left(X_{i}(s), V(s)\right) d B_{i}(s)+\int_{0}^{t} c\left(X_{i}(s), V(s)\right) d s \\
& +\int_{U \times[0, t]} \alpha\left(X_{i}(s), V(s), u\right) W(d u d s)
\end{aligned}
$$

and

$$
\begin{aligned}
A_{i}(t)= & A_{i}(0)+\int_{0}^{t} A_{i}(s) \gamma^{T}\left(X_{i}(s), V(s)\right) d B_{i}(s)+\int_{0}^{t} A_{i}(s) d\left(X_{i}(s), V(s)\right) d s \\
& +\int_{U \times[0, t]} A_{i}(s) \beta\left(X_{i}(s), V(s), u\right) W(d u d s),
\end{aligned}
$$

where the $B_{i}$ are independent, standard $\mathbb{R}^{d}$-valued Brownian motions, independent of $W$, and $\left\{\left(X_{i}(0), A_{i}(0)\right)\right\}$ is an exchangeable sequence of random variables in $\mathbb{R}^{d} \times \mathbb{R}$ that is independent of $\left\{B_{i}\right\}$ and $W$. Here $\sigma, c$ and $\gamma$ are related to $a, b, \alpha$, and $\beta$ by

$$
a(x, v)=\sigma(x, v) \sigma^{T}(x, v)+\int_{U} \alpha(x, v, u) \alpha^{T}(x, v, u) \mu(d u)
$$

and

$$
b(x, v)=c(x, v)+\sigma(x, v) \gamma(x, v)+\int_{U} \beta(x, v, u) \alpha(x, v, u) \mu(d u)
$$

The representation of the solution given by (1.3) suggests that the solution can be approximated by the weighted empirical measure

$$
V^{n}(t)=\frac{1}{n} \sum_{i=1}^{n} A_{i}^{n}(t) \delta_{X_{i}^{n}(t)},
$$


of a finite particle system satisfying

$$
\begin{aligned}
X_{i}^{n}(t)= & X_{i}(0)+\int_{0}^{t} \sigma\left(X_{i}^{n}(s), V^{n}(s)\right) d B_{i}(s)+\int_{0}^{t} c\left(X_{i}^{n}(s), V^{n}(s)\right) d s \\
& +\int_{U \times[0, t]} \alpha\left(X_{i}^{n}(s), V^{n}(s), u\right) W(d u d s) \\
A_{i}^{n}(t)= & A_{i}(0)+\int_{0}^{t} A_{i}^{n}(s) \gamma^{T}\left(X_{i}^{n}(s), V^{n}(s)\right) d B_{i}(s)+\int_{0}^{t} A_{i}^{n}(s) d\left(X_{i}^{n}(s), V^{n}(s)\right) d s \\
& +\int_{U \times[0, t]} A_{i}^{n}(s) \beta\left(X_{i}^{n}(s), V^{n}(s), u\right) W(d u d s),
\end{aligned}
$$

for $i=1,2, \cdots, n$.

In [27], it was shown that for an appropriate metric $\widetilde{\rho}$ on $\mathcal{M}\left(\mathbb{R}^{d}\right)$, $\left\{\sqrt{n} \widetilde{\rho}\left(V^{n}(t), V(t)\right)\right\}_{n \geq 1}$ is stochastically bounded, that is, for each $\epsilon>0$, there is a constant $K_{\epsilon}$ such that

$$
\sup _{n} \mathbb{P}\left(\sqrt{n} \widetilde{\rho}\left(V^{n}(t), V(t)\right)>K_{\epsilon}\right)<\epsilon
$$

From (1.9), we see that the convergence rate has an upper bound of the order of $\frac{1}{\sqrt{n}}$. A natural question to ask is whether $\frac{1}{\sqrt{n}}$ is the right order. To this end, we study the convergence of the process $S_{n}(t)=\sqrt{n}\left(V^{n}(t)-V(t)\right)$ and show that for an appropriate space $\Phi_{-\kappa}$ of distributions, $S_{n}$ converges in distribution in $C_{\Phi_{-\kappa}}[0, \infty)$. We characterize the limit $S$ as the unique solution of a stochastic evolution equation of the form

$$
\begin{array}{r}
\langle\phi, S(t)\rangle=\langle\phi, S(0)\rangle+\langle\phi, M(t)\rangle+\int_{0}^{t}\left\langle F_{1}(V(s)) \phi, S(s)\right\rangle d s \\
+\int_{U \times[0, t]}\left\langle F_{2}(V(s), u) \phi, S(s)\right\rangle W(d u d s),
\end{array}
$$

where $F_{1}$ and $F_{2}$ are linear in $\phi$ and $M$ is a distribution-valued martingale. $F_{1}$ and $F_{2}$ are defined in Assumption (S6) in Section 4 in terms of appropriate differentials of the coefficients of (1.2), reflecting the fact that we are rescaling the deviation of $V^{n}$ from $V$.

This type of problem has been studied by various authors in the McKean-Vlasov setting, that is, $\alpha=0$ and $A_{i}^{n}(t) \equiv 1$ (cf. Hitsuda and Mitoma [19] and the references therein). Comparing the present results with those of [19], here the process $V$ is not deterministic and the process $S$ is not Gaussian. In [19], the limit $S$ is characterized by its covariance structure which, because $S$ is Gaussian, uniquely determines its distribution. A stochastic evolution equation is also derived in that paper. The uniqueness of the solution to that stochastic evolution equation is proved by Mitoma [33].

Another new feature in this paper is that the driving martingale $M$ in the evolution equation (1.10) is not Gaussian and has to be defined by the particle system $\left\{X_{i}, A_{i}, V\right\}$ itself. The main difficulty in establishing the uniqueness of the solution of (1.10) comes from the addition of the last term in (1.10) which does not appear in [19] and [33]. 
Limits of empirical measure processes for systems of interacting diffusions have been studied by various authors (see, for example, Chiang, Kallianpur and Sundar [4], Graham [18], Kallianpur and Xiong [22], Méléard [31], and Morien [34]) since the pioneering work by McKean [30]. Typically, the driving processes in the models are assumed to be independent, and the limit is then a deterministic, measure-valued function.

Florchinger and Le Gland [14] consider particle approximations for stochastic partial differential equations in a setting that, in the notation above, corresponds to taking $\gamma=\sigma=0$ and the other coefficients independent of $V$. Florchinger and Le Gland were motivated by approximations to the Zakai equation of nonlinear filtering. Del Moral [10] specifically studies this example. Kotelenez [23] introduces a model of $n$-particles with the same driving process for each particle and studies the empirical process as the solution of an SPDE. His model corresponds to taking $\gamma=\sigma=d=\beta=0$, but the other coefficients are allowed to depend on $V$. In particular, the weights $A_{i}$ are constants. Dawson and Vaillancourt [9] consider a model given as a solution of a martingale problem that corresponds to taking $A_{i}(t) \equiv 1$ in the current model. Bernard, Talay, and Tubaro [1] consider a system with time-varying weights and a deterministic limit.

The paper is organized as follows: In the next section, we derive key estimates on the magnitude of the $A_{i}^{n}$ and on the error in the approximation of $\left(X_{i}, A_{i}\right)$ by $\left(X_{i}^{n}, A_{i}^{n}\right)$. In Section 3, we prove that $\left\{S_{n}\right\}$ is a tight sequence of $\Phi^{\prime}$-valued processes ( $\Phi^{\prime}$ being a conuclear space defined later). Then, in Section 4 , we show that the limit $S$ of $\left\{S_{n}\right\}$ is the unique solution of (1.10).

If one wants to use the finite system to simulate the solution of the SPDE, then the finite system must also be approximated. The simplest approach is to use an Euler approximation. In the last section of this paper, we analyze this approximation in the simplest setting, assuming that $W$ is a one-dimensional Brownian motion (that is, $U$ consists of a single point). Letting $V^{n, 1 / n}$ denote the weighted empirical measure for the Euler scheme approximating the finite system (cf. (5.1-5.3)), we consider the process $\widetilde{S}_{n}(t)=\sqrt{n}\left(V^{n, 1 / n}(t)-V^{n}(t)\right)$. We prove tightness for $\left\{\widetilde{S}_{n}\right\}$ and characterize its limit as the unique solution of another stochastic evolution equation. Finally, we combine the two parts and derive a stochastic evolution equation for the limit of $\sqrt{n}\left(V^{n, 1 / n}-V\right)$.

\section{Preliminaries}

In this section, we state the main results of [26] and [27] needed in the present paper for the convenience of the reader. The following assumptions were made in [26] for the existence and uniqueness of solutions of the SPDE (1.1).

(S1) There exists a constant $K$ such that for each $x \in \mathbb{R}^{d}, \nu \in \mathcal{M}\left(\mathbb{R}^{d}\right)$

$$
\begin{aligned}
& |\sigma(x, \nu)|^{2}+|c(x, \nu)|^{2}+\int_{U}|\alpha(x, \nu, u)|^{2} \mu(d u) \\
& +|\gamma(x, \nu)|^{2}+|d(x, \nu)|^{2}+\int_{U} \beta(x, \nu, u)^{2} \mu(d u) \leq K^{2} .
\end{aligned}
$$

(S2) For each $x_{1}, x_{2} \in \mathbb{R}^{d}, \nu_{1}, \nu_{2} \in \mathcal{M}\left(\mathbb{R}^{d}\right)$

$$
\left|\sigma\left(x_{1}, \nu_{1}\right)-\sigma\left(x_{2}, \nu_{2}\right)\right|^{2}+\left|c\left(x_{1}, \nu_{1}\right)-c\left(x_{2}, \nu_{2}\right)\right|^{2}
$$




$$
\begin{aligned}
& \quad+\left|\gamma\left(x_{1}, \nu_{1}\right)-\gamma\left(x_{1}, \nu_{1}\right)\right|^{2}+\int_{U}\left|\alpha\left(x_{1}, \nu_{1}, u\right)-\alpha\left(x_{2}, \nu_{2}, u\right)\right|^{2} \mu(d u) \\
& +\left|d\left(x_{1}, \nu_{1}\right)-d\left(x_{2}, \nu_{2}\right)\right|^{2}+\int_{U}\left|\beta\left(x_{1}, \nu_{1}, u\right)-\beta\left(x_{2}, \nu_{2}, u\right)\right|^{2} \mu(d u) \\
& \leq K^{2}\left(\left|x_{1}-x_{2}\right|^{2}+\rho\left(\nu_{1}, \nu_{2}\right)^{2}\right),
\end{aligned}
$$

where

$$
\rho\left(\nu_{1}, \nu_{2}\right)=\sup \left\{\left|\left\langle\phi, \nu_{1}\right\rangle-\left\langle\phi, \nu_{2}\right\rangle\right|: \phi \in \mathbb{B}_{1}\right\}
$$

and

$$
\mathbb{B}_{1}=\left\{\phi:|\phi(x)-\phi(y)| \leq|x-y|,|\phi(x)| \leq 1, \forall x, y \in \mathbb{R}^{d}\right\} .
$$

By the same proof as in Proposition 2.1 of [26], we have the following result.

Proposition 2.1. Suppose that Assumption (S1) holds and $p$ is a positive number. i) If

$$
\mathbb{E} e^{p\left|X_{1}(0)\right|}<\infty,
$$

then

$$
\sup _{1 \leq n \leq \infty} \mathbb{E} \sup _{0 \leq s \leq T} e^{p\left|X_{i}^{n}(s)\right|}<\infty
$$

ii) If

$$
\mathbb{E}\left|A_{1}(0)\right|^{p}<\infty,
$$

then

$$
\sup _{1 \leq n \leq \infty} \mathbb{E} \sup _{0 \leq s \leq T}\left|A_{i}^{n}(s)\right|^{p}<\infty .
$$

iii) If

$$
\mathbb{E}\left|A_{1}(0)\right| e^{r\left|X_{1}(0)\right|}<\infty,
$$

then

$$
\sup _{1 \leq n \leq \infty} \mathbb{E} \sup _{0 \leq s \leq T}\left|A_{i}^{n}(s)\right| e^{r\left|X_{i}^{n}(s)\right|}<\infty
$$

REMARK 2.2. If (2.1) and (2.3) hold, then (2.5) holds with $r=p-1$.

A weaker form of the following assumption was used in [27].

(S3) There exist constants $\lambda>1$ and $K>0$ such that for any iid sequence $\left(\xi_{i}, \eta_{i}\right), i=1,2, \cdots$ and $x \in \mathbb{R}^{d}$,

$$
\mathbb{E}\left|\sigma\left(x, \frac{1}{n} \sum_{i=1}^{n} \xi_{i} \delta_{\eta_{i}}\right)-\sigma(x, \mu)\right|^{2 \lambda} \leq \frac{K \mathbb{E} \xi_{1}^{2 \lambda}}{n^{\lambda}},
$$

where $\mu(\cdot)=\mathbb{E}\left[\xi_{1} 1_{\left.\eta_{1} \in \cdot\right]}\right]$, and a similar inequality holds for the other coefficients. 
REMARK 2.3. If $\sigma(x, \mu)=\int \sigma_{1}(x, y) \mu(d y)$ or $\sigma(x, \mu)=\sigma_{1}(x)$, then (S3) usually holds. For example, if $\left|\sigma_{1}(x, y)\right| \leq K$, then (S3) holds.

The following estimate is the key for the proof of the tightness of $\left\{S_{n}\right\}$.

THEOREM 2.4. Under the assumptions (S1)-(S3), there exists a constant $c_{1}(T, m)$ such that

$$
\begin{aligned}
& \operatorname{Esup}_{t \leq T}\left(\left|X_{i}^{n}\left(t \wedge \eta_{m}^{n}\right)-X_{i}\left(t \wedge \eta_{m}^{n}\right)\right|^{2 \lambda}+\left(\frac{1}{n} \sum_{j=1}^{n}\left|A_{j}^{n}\left(t \wedge \eta_{m}^{n}\right)-A_{j}\left(t \wedge \eta_{m}^{n}\right)\right|^{\lambda}\right)^{2}\right) \\
& \quad \leq \frac{c_{1}(T, m)}{n^{\lambda}}
\end{aligned}
$$

where

$$
\eta_{m}^{n}=\inf \left\{t: \frac{1}{n} \sum_{i=1}^{n} A_{i}^{n}(t)^{2}>m^{2} \text { or } \lim _{k \rightarrow \infty} \frac{1}{k} \sum_{i=1}^{k} A_{i}(t)^{2}>m^{2}\right\} .
$$

Proof: By Doob's inequality and Holder's inequality, we have

$$
\begin{aligned}
\mathbb{E} \sup _{r \leq t} \mid X_{i}^{n} & \left(r \wedge \eta_{m}^{n}\right)-\left.X_{i}\left(r \wedge \eta_{m}^{n}\right)\right|^{2 \lambda} \\
\leq 3^{2 \lambda} & \left(\frac{2 \lambda}{2 \lambda-1}\right)^{2 \lambda} \mathbb{E} \int_{0}^{t}\left|\sigma\left(X_{i}^{n}(s), V^{n}(s)\right)-\sigma\left(X_{i}(s), V(s)\right)\right|^{2 \lambda} 1_{s \leq \eta_{m}^{n}} d s t^{\lambda-1} \\
& +3^{2 \lambda} t^{2 \lambda-1} \mathbb{E} \int_{0}^{t}\left|c\left(X_{i}^{n}(s), V^{n}(s)\right)-c\left(X_{i}(s), V(s)\right)\right|^{2 \lambda} 1_{s \leq \eta_{m}^{n}} d s \\
& +3^{2 \lambda}\left(\frac{2 \lambda}{2 \lambda-1}\right)^{2 \lambda} t^{\lambda-1} \\
& \times \mathbb{E} \int_{0}^{t}\left(\int_{U}\left|\alpha\left(X_{i}^{n}(s), V^{n}(s), u\right)-\alpha\left(X_{i}(s), V(s), u\right)\right|^{2} \mu(d u)\right)^{\lambda} 1_{s \leq \eta_{m}^{n}} d s .
\end{aligned}
$$

Let

$$
\widetilde{V}^{n}(t)=\frac{1}{n} \sum_{i=1}^{n} A_{i}(t) \delta_{X_{i}(t)} \quad \text { and } \quad \widetilde{V}_{i}^{n}(t)=\frac{1}{n-1} \sum_{j=1, j \neq i}^{n} A_{j}(t) \delta_{X_{j}(t)} .
$$

Then

$$
\begin{aligned}
& \mathbb{E}\left|\sigma\left(X_{i}^{n}(s), V^{n}(s)\right)-\sigma\left(X_{i}(s), V(s)\right)\right|^{2 \lambda} 1_{s \leq \eta_{m}^{n}} \\
& \leq 3^{2 \lambda} \mathbb{E}\left|\sigma\left(X_{i}^{n}(s), V^{n}(s)\right)-\sigma\left(X_{i}(s), \widetilde{V}^{n}(s)\right)\right|^{2 \lambda} 1_{s \leq \eta_{m}^{n}} \\
& \quad+3^{2 \lambda} \mathbb{E}\left|\sigma\left(X_{i}(s), \widetilde{V}^{n}(s)\right)-\sigma\left(X_{i}(s), \widetilde{V}_{i}^{n}(s)\right)\right|^{2 \lambda} \\
& \quad+3^{2 \lambda} \mathbb{E}\left|\sigma\left(X_{i}(s), \widetilde{V}_{i}^{n}(s)\right)-\sigma\left(X_{i}(s), V(s)\right)\right|^{2 \lambda} 1_{s \leq \eta_{m}^{n}} \\
& \leq 3^{2 \lambda} K^{2 \lambda} \mathbb{E}\left(\left|X_{i}^{n}(s)-X_{i}(s)\right|^{2}+\rho\left(V^{n}(s), \widetilde{V}^{n}(s)\right)^{2}\right)^{\lambda} 1_{s \leq \eta_{m}^{n}} \\
& \quad+3^{2 \lambda} K^{2 \lambda} \mathbb{E} \rho\left(\widetilde{V}^{n}(s), \widetilde{V}_{i}^{n}(s)\right)^{2 \lambda} 1_{s \leq \eta_{m}^{n}} \\
& \quad+3^{2 \lambda} \mathbb{E}\left[\mathbb{E}\left[\left|\sigma\left(X_{i}(s), \frac{1}{n-1} \sum_{j=1, j \neq i}^{n} A_{j}(s) \delta_{X_{j}(s)}\right)-\sigma\left(X_{i}(s), V(s)\right)^{2 \lambda}\right| \mid W, X_{i}\right]\right] .
\end{aligned}
$$


Note that, similar to the arguments in the proof of Theorem 2.1 in [26], we have

$$
\begin{aligned}
& \rho\left(V^{n}(s), \widetilde{V}^{n}(s)\right) \\
& \quad \leq \frac{1}{n} \sum_{j=1}^{n} A_{j}^{n}(s)\left|X_{j}^{n}(s)-X_{j}(s)\right|+\frac{1}{n} \sum_{j=1}^{n}\left|A_{j}^{n}(s)-A_{j}(s)\right| \\
& \quad \leq\left(\frac{1}{n} \sum_{j=1}^{n} A_{j}^{n}(s)^{2}\right)^{1 / 2}\left(\frac{1}{n} \sum_{j=1}^{n}\left|X_{j}^{n}(s)-X_{j}(s)\right|^{2}\right)^{1 / 2}+\frac{1}{n} \sum_{j=1}^{n}\left|A_{j}^{n}(s)-A_{j}(s)\right| .
\end{aligned}
$$

Similarly,

$$
\rho\left(\widetilde{V}^{n}(s), \widetilde{V}_{i}^{n}(s)\right) \leq \frac{1}{n} A_{i}(s)+\frac{1}{n(n-1)} \sum_{j=1}^{n} A_{j}(s) .
$$

Let

$$
f_{m}^{n}(t)=\mathbb{E} \sup _{r \leq t}\left|X_{i}^{n}\left(r \wedge \eta_{m}^{n}\right)-X_{i}\left(r \wedge \eta_{m}^{n}\right)\right|^{2 \lambda}
$$

and

$$
g_{m}^{n}(t)=\mathbb{E} \sup _{r \leq t}\left(\frac{1}{n} \sum_{j=1}^{n}\left|A_{j}^{n}\left(r \wedge \eta_{m}^{n}\right)-A_{j}\left(r \wedge \eta_{m}^{n}\right)\right|^{\lambda}\right)^{2}
$$

Then, for the right hand side of (2.8),

$$
\begin{aligned}
1 \text { st term } \leq & 3^{2 \lambda} K^{2 \lambda} 2^{\lambda}\left(\mathbb{E}\left|X_{i}^{n}\left(s \wedge \eta_{m}^{n}\right)-X_{i}\left(s \wedge \eta_{m}^{n}\right)\right|^{2 \lambda}\right. \\
& \left.+2^{2 \lambda} m^{2 \lambda} \mathbb{E} \frac{1}{n} \sum_{j=1}^{n}\left|X_{j}^{n}\left(s \wedge \eta_{m}^{n}\right)-X_{j}\left(s \wedge \eta_{m}^{n}\right)\right|^{2 \lambda}+2^{2 \lambda} g_{m}^{n}(s)\right) \\
\leq & 18^{\lambda} K^{2 \lambda}\left(f_{m}^{n}(s)+4^{\lambda} m^{2 \lambda} f_{m}^{n}(s)+4^{\lambda} g_{m}^{n}(s)\right)
\end{aligned}
$$

and

$$
\text { 2nd term } \leq 3^{2 \lambda} K^{2 \lambda} 2^{2 \lambda}\left(\frac{1}{n^{2 \lambda}} \mathbb{E} \sup _{r \leq t}\left|A_{i}(r)\right|^{2 \lambda}+\frac{1}{(n-1)^{2 \lambda}} m^{2 \lambda}\right) .
$$

Since, conditioning on $\left(W, X_{i}\right),\left(A_{j}, X_{j}\right), j \neq i$, are $i i d$, we have

$$
\text { 3rd term } \leq 3^{2 \lambda} \mathbb{E}\left(\frac{K^{2 \lambda}}{(n-1)^{\lambda}} \mathbb{E}\left(A_{1}(s)^{2 \lambda} \mid W, X_{i}\right)\right)=\frac{3^{2 \lambda} K^{2 \lambda}}{(n-1)^{\lambda}} \mathbb{E} A_{1}(s)^{2 \lambda} \text {. }
$$

Hence, the first term on the right hand side of (2.7) is dominated by

$$
\begin{aligned}
& \left(\frac{6 \lambda}{2 \lambda-1}\right)^{2 \lambda} T^{\lambda-1} \int_{0}^{t} 18^{\lambda} K^{2 \lambda}\left(\left(1+2^{\lambda} m^{2 \lambda}\right) f_{m}^{n}(s)+2^{\lambda} g_{m}^{n}(s)\right) d s \\
& +\left(\frac{6 \lambda}{2 \lambda-1}\right)^{2 \lambda}\left[3^{2 \lambda} K^{2 \lambda} 2^{2 \lambda}\left(\frac{1}{n^{2 \lambda}} \mathbb{E}_{r \leq t}\left|A_{i}(r)\right|^{2 \lambda}+\frac{1}{(n-1)^{2 \lambda}} m^{2 \lambda}\right)\right. \\
& \left.+\frac{3^{2 \lambda} K^{2 \lambda}}{(n-1)^{\lambda}} \mathbb{E} A_{1}(s)^{2 \lambda}\right] T^{\lambda} .
\end{aligned}
$$


Similar estimates hold for other terms on the right hand side of (2.7). Therefore, there exist constants $c_{2}(T, m)$ and $c_{3}(T, m)$ such that

$$
f_{m}^{n}(t) \leq c_{2}(T, m) \int_{0}^{t}\left(f_{m}^{n}(s)+g_{m}^{n}(s)\right) d s+\frac{c_{3}(T, m)}{n^{\lambda}} .
$$

By similar arguments as in (2.7) of [26] and (2.10) above, we have

$$
g_{m}^{n}(t) \leq c_{4}(T, m) \int_{0}^{t}\left(f_{m}^{n}(s)+g_{m}^{n}(s)\right) d s+\frac{c_{5}(T, m)}{n^{\lambda}} .
$$

Therefore

$$
f_{m}^{n}(t)+g_{m}^{n}(t) \leq\left(c_{2}+c_{4}\right) \int_{0}^{t}\left(f_{m}^{n}(s)+g_{m}^{n}(s)\right) d s+\frac{c_{3}+c_{5}}{n^{\lambda}} .
$$

By Gronwall's inequality, we have

$$
f_{m}^{n}(t)+g_{m}^{n}(t) \leq \frac{c_{1}}{n^{\lambda}}
$$

by taking $c_{1}=\frac{e^{\left(c_{2}+c_{4}\right) T}\left(c_{3}+c_{5}\right)}{c_{2}+c_{4}}$.

\section{Tightness}

In this section, we prove tightness for $\left\{S_{n}\right\}$ in an appropriate space. For simplicity of notation, we restrict our calculations to space dimension $d=1$ in the rest of this paper.

As in Hitsuda and Mitoma [19], we use the modified Schwartz space $\Phi$. Let $\rho(x)=C \exp \left(-1 /\left(1-|x|^{2}\right)\right) 1_{|x|<1}$ where $C$ is a constant such that $\int \rho(x) d x=1$. Let

$$
\psi(x)=\int e^{-|y|} \rho(x-y) d y .
$$

Then for any integer $k$, we have $\left|e^{(k)}(x)\right| \leq c_{6}(k)\left(1+e^{|x|}\right)$. Let

$$
\Phi=\{\phi: \phi \psi \in \mathcal{S}\}
$$

where $\mathcal{S}$ is the Schwartz space. For $\kappa=0,1,2, \ldots$, define

$$
\|\phi\|_{\kappa}^{2}=\sum_{0 \leq k \leq \kappa} \int_{\mathbb{R}}\left(1+|x|^{2}\right)^{2 \kappa}\left|\frac{d^{k}}{d x^{k}}(\phi(x) \psi(x))\right|^{2} d x .
$$

Let $\Phi_{\kappa}$ be the completion of $\Phi$ with respect to $\|\cdot\|_{\kappa}$. Then $\Phi_{\kappa}$ is a Hilbert space with inner product

$$
\left\langle\phi_{1}, \phi_{2}\right\rangle_{\kappa}=\sum_{0 \leq k \leq \kappa} \int_{\mathbb{R}}\left(1+|x|^{2}\right)^{2 \kappa}\left(\frac{d^{k}}{d x^{k}}\left(\phi_{1}(x) \psi(x)\right)\right)\left(\frac{d^{k}}{d x^{k}}\left(\phi_{2}(x) \psi(x)\right)\right) d x .
$$

Note that $\Phi_{\kappa} \supset \Phi_{\kappa+1}$ and that $\Phi_{0}$ is $L^{2}\left(\mu_{\psi}\right)$, where $\mu_{\psi}(d x)=\psi^{2}(x) d x$. For $\widehat{\phi} \in \Phi_{0}$ and $\phi \in \Phi_{\kappa}$,

$$
\langle\widehat{\phi}, \phi\rangle \equiv\langle\widehat{\phi}, \phi\rangle_{0}=\int_{\mathbb{R}} \widehat{\phi}(x) \phi(x) \psi^{2}(x) d x
$$


defines a continuous linear functional on $\Phi_{\kappa}$ with norm

$$
\|\widehat{\phi}\|_{-\kappa}=\sup _{\phi \in \Phi_{\kappa}} \frac{|\langle\widehat{\phi}, \phi\rangle|}{\|\phi\|_{\kappa}},
$$

and we let $\Phi_{-\kappa}$ denote the completion of $\Phi_{0}$ with respect to this norm. Then $\Phi_{-\kappa}$ is a representation of the dual of $\Phi_{\kappa}$. If $\left\{\phi_{j}^{\kappa}\right\}$ is a complete, orthonormal system for $\Phi_{\kappa}$, then the inner product for $\Phi_{-\kappa}$ can be written as

$$
\left\langle\widehat{\phi}_{1}, \widehat{\phi}_{2}\right\rangle_{-\kappa}=\sum_{j=1}^{\infty}\left\langle\widehat{\phi}_{1}, \phi_{j}^{\kappa}\right\rangle\left\langle\widehat{\phi}_{2}, \phi_{j}^{\kappa}\right\rangle
$$

By a slight modification of Theorem 7, page 82, of [17], these norms determine a nuclear space, so in particular, for each $\kappa$ there exists a $\kappa^{\prime}>\kappa$ such that the embedding $T_{\kappa}^{\kappa^{\prime}}: \Phi_{\kappa^{\prime}} \rightarrow \Phi_{\kappa}$ is a Hilbert-Schmidt operator. The adjoint $T_{\kappa}^{\kappa^{\prime} *}: \Phi_{-\kappa} \rightarrow \Phi_{-\kappa^{\prime}}$ is also Hilbert-Schmidt. $\Phi^{\prime}=\cup_{k=0}^{\infty} \Phi_{-k}$ gives a representation of the dual of $\Phi$. (See [17], page 59.) We prove tightness for $\left\{S_{n}\right\}$ in $C_{\Phi_{-\kappa}}[0, \infty)$ for an appropriate $\kappa$.

THEOREM 3.1. Suppose that (S1)-(S3) hold and that (2.1) and (2.3) hold for

$$
p=\max (4 \lambda, \lambda /(\lambda-1)) \text {. }
$$

Then there exists $\kappa$ such that $\left\{S_{n}\right\}$ is tight in $C_{\Phi_{-\kappa}}[0, \infty)$.

Proof: Let

$$
\eta_{m}^{n, p}=\inf \left\{t: \frac{1}{n} \sum_{i=1}^{n} A_{i}^{n}(t)^{p} \geq m^{p} \text { or } \frac{1}{n} \sum_{i=1}^{n}\left(1+e^{p\left|X_{i}^{n}(t)\right|} \vee e^{p\left|X_{i}(t)\right|}\right) \geq m^{p}\right\}
$$

Then for $T>0$, we have

$$
\sup _{n} \mathbb{P}\left(\eta_{m}^{n, p} \leq T\right) \leq \frac{2}{m^{p}} \sup _{1 \leq n \leq \infty}\left(1+\mathbb{E} \sup _{0 \leq s \leq T} A_{i}^{n}(s)^{p}+\mathbb{E} \sup _{0 \leq s \leq T} e^{p\left|X_{i}^{n}(s)\right|}\right),
$$

and since by Proposition 2.1, for each $T>0$, the right side goes to zero as $m \rightarrow \infty$, it is enough to prove tightness for $\left\{S_{n}\left(\cdot \wedge \eta_{m}^{n, p}\right)\right\}$.

By Itô's formula, we have

$$
\begin{aligned}
\left\langle\phi, V^{n}(t)\right\rangle & -\left\langle\phi, V^{n}(0)\right\rangle \\
= & \frac{1}{n} \sum_{i=1}^{n} \int_{0}^{t} A_{i}^{n}(s)\left(\phi\left(X_{i}^{n}(s)\right) \gamma\left(X_{i}^{n}(s), V^{n}(s)\right)\right. \\
& \left.+\phi^{\prime}\left(X_{i}^{n}(s)\right) \sigma\left(X_{i}^{n}(s), V^{n}(s)\right)\right) d B_{i}(s) \\
& +\int_{0}^{t}\left\langle\phi d\left(\cdot, V^{n}(s)\right)+L\left(V^{n}(s)\right) \phi, V^{n}(s)\right\rangle d s \\
& +\int_{U \times[0, t]}\left\langle\phi \beta\left(\cdot, V^{n}(s), u\right)+\phi^{\prime} \alpha\left(\cdot, V^{n}(s), u\right), V^{n}(s)\right\rangle W(d u d s),
\end{aligned}
$$

where

$$
L(v) \phi(x)=\frac{1}{2} a(x, v) \phi^{\prime \prime}(x)+b(x, v) \phi^{\prime}(x) .
$$


Hence, by (1.2) and (3.2), we have

$$
\begin{aligned}
& \left\langle\phi, S_{n}(t)-S_{n}(0)\right\rangle \\
& =\frac{1}{\sqrt{n}} \sum_{i=1}^{n} \int_{0}^{t} A_{i}^{n}(s)\left(\phi\left(X_{i}^{n}(s)\right) \gamma\left(X_{i}^{n}(s), V^{n}(s)\right)\right. \\
& \left.\quad+\phi^{\prime}\left(X_{i}^{n}(s)\right) \sigma\left(X_{i}^{n}(s), V^{n}(s)\right)\right) d B_{i}(s) \\
& +\int_{0}^{t} \sqrt{n}\left(\left\langle\phi d\left(\cdot, V^{n}(s)\right)+L\left(V^{n}(s)\right) \phi, V^{n}(s)\right\rangle\right. \\
& \quad-\langle\phi d(\cdot, V(s))+L(V(s)) \phi, V(s)\rangle) d s \\
& \quad \int_{U \times[0, t]} \sqrt{n}\left(\left\langle\phi \beta\left(\cdot, V^{n}(s), u\right)+\phi^{\prime} \alpha\left(\cdot, V^{n}(s), u\right), V^{n}(s)\right\rangle\right. \\
& \left.-\left\langle\phi \beta(\cdot, V(s), u)+\phi^{\prime} \alpha(\cdot, V(s), u), V(s)\right\rangle\right) W(d u d s) .
\end{aligned}
$$

Note that $\left\langle\phi, S_{n}(t)\right\rangle=M_{\phi}^{1, n}(t)+A_{\phi}^{n}(t)+M_{\phi}^{2, n}(t)$ is a semimartingale with respect to the filtration $\left\{\mathcal{F}_{t}\right\}$ generated by $W$ and the $B_{i}$. Setting

$$
\begin{aligned}
G_{\phi}^{n}(s, u)=\sqrt{n}\left(\left\langle\phi \beta\left(\cdot, V^{n}(s), u\right)+\phi^{\prime} \alpha\left(\cdot, V^{n}(s), u\right), V^{n}(s)\right\rangle\right. \\
\left.-\left\langle\phi \beta(\cdot, V(s), u)+\phi^{\prime} \alpha(\cdot, V(s), u), V(s)\right\rangle\right),
\end{aligned}
$$

we have

$$
\begin{aligned}
& {\left[M_{\phi}^{1, n}\right]_{t}} \\
& \quad=\frac{1}{n} \sum_{i=1}^{n} \int_{0}^{t} A_{i}^{n}(s)^{2}\left(\phi\left(X_{i}^{n}(s)\right) \gamma\left(X_{i}^{n}(s), V^{n}(s)\right)+\phi^{\prime}\left(X_{i}^{n}(s)\right) \sigma\left(X_{i}^{n}(s), V^{n}(s)\right)\right)^{2} d s
\end{aligned}
$$

and

$$
\left[M^{2, n}\right]_{t}=\int_{0}^{t} \int_{U} G_{\phi}^{n}(s, u)^{2} \mu(d u) d t
$$

Let

$$
\begin{aligned}
& H_{\phi}^{1, n}(s)=\frac{1}{n} \sum_{i=1}^{n} A_{i}^{n}(s)^{2}\left(\phi\left(X_{i}^{n}(s)\right) \gamma\left(X_{i}^{n}(s), V^{n}(s)\right)+\phi^{\prime}\left(X_{i}^{n}(s)\right) \sigma\left(X_{i}^{n}(s), V^{n}(s)\right)\right)^{2} \\
& H_{\phi}^{2, n}(s)=\int_{U} G_{\phi}^{n}(s, u)^{2} \mu(d u) \\
& H_{\phi}^{3, n}(s)=n\left(\left\langle\phi d\left(\cdot, V^{n}(s)\right)+L\left(V^{n}(s)\right) \phi, V^{n}(s)\right\rangle-\langle\phi d(\cdot, V(s))+L(V(s)) \phi, V(s)\rangle\right)^{2} .
\end{aligned}
$$

It follows, for example, that for $t, h>0$, we have

$$
\mathbb{E}\left[\left\langle\phi, S_{n}(t+h)-S_{n}(t)\right\rangle^{2} \mid \mathcal{F}_{t}\right] \leq \mathbb{E}\left[\int_{t}^{t+h} 3\left(H_{\phi}^{1, n}(s)+H_{\phi}^{2, n}(s)+h H_{\phi}^{3, n}(s)\right) d s \mid \mathcal{F}_{t}\right]
$$

and, applying Doob's inequality,

$$
\mathbb{E}\left[\sup _{s \leq t}\left\langle\phi, S_{n}(s)-S_{n}(0)\right\rangle^{2}\right] \leq \mathbb{E}\left[\int_{0}^{t} 12\left(H_{\phi}^{1, n}(s)+H_{\phi}^{2, n}(s)+t H_{\phi}^{3, n}(s)\right) d s\right] .
$$


We need to estimate each of the $H_{\phi}^{k, n}$.

Let $\widetilde{\phi}=\phi \psi$ and $|\widetilde{\phi}|_{\kappa}=\sup _{x, 0 \leq k \leq \kappa}\left|\left(d^{k} / d x^{k}\right) \widetilde{\phi}(x)\right|$. Then $|\widetilde{\phi}|_{\kappa} \leq \mathrm{const}\|\phi\|_{\kappa+1}$. It is easy to see that there exists a constant $c_{7}$ such that

$$
|\phi(x)| \leq c_{7}|\widetilde{\phi}|_{0} e^{|x|}
$$

and

$$
|\phi(x)|+\left|\phi^{\prime}(x)\right| \leq c_{7}|\widetilde{\phi}|_{1} e^{|x|} .
$$

Hence

$$
\begin{aligned}
\mathbb{E} \mid \frac{1}{n} & \left.\sum_{i=1}^{n} A_{i}^{n}(s)^{2}\left(\phi\left(X_{i}^{n}(s)\right) \gamma\left(X_{i}^{n}(s), V^{n}(s)\right)+\phi^{\prime}\left(X_{i}^{n}(s)\right) \sigma\left(X_{i}^{n}(s), V^{n}(s)\right)\right)^{2}\right|^{\lambda} 1_{\left\{\eta_{m}^{n, p} \geq s\right\}} \\
& \leq\left.\left.\mathbb{E}\left|\frac{1}{n} \sum_{i=1}^{n} A_{i}^{n}(s)^{2} K^{2} c_{7} e^{2\left|X_{i}^{n}(s)\right|}\right| \widetilde{\phi}\right|_{1} ^{2}\right|^{\lambda} 1_{\left\{\eta_{m}^{n, p} \geq s\right\}} \\
& \leq K^{2 \lambda} c_{7}^{\lambda}|\widetilde{\phi}|_{1}^{2 \lambda} \mathbb{E}\left|\frac{1}{n} \sum_{i=1}^{n} A_{i}^{n}(s)^{4} \frac{1}{n} \sum_{i=1}^{n} e^{4\left|X_{i}^{n}(s)\right|}\right|^{\frac{\lambda}{2}} 1_{\left\{\eta_{m}^{n, p} \geq s\right\}} \\
& \leq K^{2 \lambda} c_{7}^{\lambda}|\widetilde{\phi}|_{1}^{2 \lambda}\left|m^{4} 2^{3} m^{4}\right|^{\frac{\lambda}{2}} \\
& \equiv c_{8}(m, \lambda, s)|\widetilde{\phi}|_{1}^{2 \lambda}
\end{aligned}
$$

and we have

$$
\mathbb{E}\left[H_{\phi}^{1, n}(s)^{\lambda} \mathbf{1}_{\left\{\eta_{m}^{n, p} \geq s\right\}}\right] \leq c_{8}(m, \lambda, s)|\widetilde{\phi}|_{1}^{2 \lambda} \leq c_{8}(m, \lambda, s)\|\phi\|_{2}^{2 \lambda} .
$$

Observing that

$$
\begin{aligned}
\left.\left.\sqrt{n}\left(\left\langle\phi d\left(\cdot, V^{n}(s)\right)\right), V^{n}(s)\right\rangle-\langle\phi d(\cdot, V(s))), V(s)\right\rangle\right) \\
\left.=\sqrt{n}\left\langle\phi d\left(\cdot, V^{n}(s)\right)\right), V^{n}(s)-\widetilde{V}^{n}(s)\right\rangle \\
\quad+\sqrt{n}\left\langle\phi\left(d\left(\cdot, V^{n}(s)\right)-d\left(\cdot, \widetilde{V}^{n}(s)\right), \widetilde{V}^{n}(s)\right\rangle\right. \\
\quad+\sqrt{n}\left\langle\phi\left(d\left(\cdot, \widetilde{V}^{n}(s)\right)-d(\cdot, V(s))\right), \widetilde{V}^{n}(s)\right\rangle \\
\quad+\sqrt{n}\left\langle\phi d(\cdot, V(s)), \widetilde{V}^{n}(s)-V(s)\right\rangle,
\end{aligned}
$$

we have

$$
\begin{aligned}
&\left|\sqrt{n}\left\langle\phi d\left(\cdot, V^{n}(s)\right)\right), V^{n}(s)-\widetilde{V}^{n}(s)\right\rangle \mid \mid \\
&=\left|\frac{1}{\sqrt{n}} \sum_{i=1}^{n}\left(A_{i}^{n}(s) \phi\left(X_{i}^{n}(s)\right) d\left(X_{i}^{n}(s), V^{n}(s)\right)-A_{i}(s) \phi\left(X_{i}(s)\right) d\left(X_{i}(s), V^{n}(s)\right)\right)\right| \\
& \leq \frac{1}{\sqrt{n}} \sum_{i=1}^{n}\left|\phi\left(X_{i}^{n}(s)\right)-\phi\left(X_{i}(s)\right)\right|\left|d\left(X_{i}^{n}(s), V^{n}(s)\right)\right| A_{i}^{n}(s) \\
& \quad+\frac{1}{\sqrt{n}} \sum_{i=1}^{n}\left|\phi\left(X_{i}(s)\right)\right|\left|d\left(X_{i}^{n}(s), V^{n}(s)\right)-d\left(X_{i}(s), V^{n}(s)\right)\right| A_{i}^{n}(s) \\
& \quad+\frac{1}{\sqrt{n}} \sum_{i=1}^{n}\left|A_{i}^{n}(s)-A_{i}(s)\right|\left|\phi\left(X_{i}(s)\right) d\left(X_{i}(s), V^{n}(s)\right)\right|
\end{aligned}
$$




$$
\begin{aligned}
& \leq \frac{K}{\sqrt{n}} \sum_{i=1}^{n}\left|\phi^{\prime}\left(\theta X_{i}^{n}(s)+(1-\theta) X_{i}(s)\right)\right|\left|X_{i}^{n}(s)-X_{i}(s)\right| A_{i}^{n}(s) \\
& \quad+\frac{K}{\sqrt{n}} \sum_{i=1}^{n}\left|\phi\left(X_{i}(s)\right)\right|\left|X_{i}^{n}(s)-X_{i}(s)\right| A_{i}^{n}(s) \\
& \quad+\frac{K}{\sqrt{n}} \sum_{i=1}^{n}\left|\phi\left(X_{i}(s)\right)\right|\left|A_{i}^{n}(s)-A_{i}(s)\right| \\
& \leq \frac{2 K}{\sqrt{n}} \sum_{i=1}^{n} c_{7}\left(e^{\left|X_{i}^{n}(s)\right|} \vee e^{\left|X_{i}(s)\right|}\right)|\widetilde{\phi}|_{1}\left|X_{i}^{n}(s)-X_{i}(s)\right| A_{i}^{n}(s) \\
& \quad+\frac{K}{\sqrt{n}} \sum_{i=1}^{n} c_{7} e^{\left|X_{i}(s)\right|}|\widetilde{\phi}|_{0}\left|A_{i}^{n}(s)-A_{i}(s)\right|,
\end{aligned}
$$

and hence

$$
\begin{aligned}
& \mathbb{E} \mid \sqrt{n}\langle\phi\left.\left.d\left(\cdot, V^{n}(s)\right)\right), V^{n}(s)-\widetilde{V}^{n}(s)\right\rangle\left.\right|^{2 \lambda} 1_{\left\{\eta_{m}^{n, p} \geq s\right\}} \\
& \leq 2^{2 \lambda-1}|\widetilde{\phi}|_{1}^{2 \lambda}\left(2 \sqrt{n} c_{7} K\right)^{2 \lambda} \mathbb{E}\left(\frac{1}{n} \sum_{i=1}^{n}\left|X_{i}^{n}(s)-X_{i}(s)\right|^{2 \lambda}\right. \\
&\left.\quad \times\left(\frac{1}{n} \sum_{i=1}^{n}\left|\left(e^{\left|X_{i}^{n}(s)\right|} \vee e^{\left|X_{i}(s)\right|}\right) A_{i}^{n}(s)\right|^{\frac{2 \lambda}{2 \lambda-1}}\right)^{2 \lambda-1} 1_{\left\{\eta_{m}^{n, p} \geq s\right\}}\right) \\
& \quad+2^{2 \lambda-1}|\widetilde{\phi}|_{0}^{2 \lambda} n^{\lambda} K^{2 \lambda} c_{7}^{2 \lambda} \mathbb{E}\left(\left(\frac{1}{n} \sum_{i=1}^{n}\left|A_{i}^{n}(s)-A_{i}(s)\right|^{\lambda}\right)^{2}\right. \\
&\left.\quad \times\left(\frac{1}{n} \sum_{i=1}^{n} e^{\frac{\lambda}{\lambda-1}\left|X_{i}(s)\right|}\right)^{\lambda-1} 1_{\left\{\eta_{m}^{n, p} \geq s\right\}}\right) \\
& \leq c_{9}(m, \lambda, s)|\widetilde{\phi}|_{1}^{2 \lambda} .
\end{aligned}
$$

As

$$
\begin{aligned}
\mid \sqrt{n} & \left\langle\phi\left(d\left(\cdot, V^{n}(s)\right)-d\left(\cdot, \widetilde{V}^{n}(s)\right), \widetilde{V}^{n}(s)\right\rangle\right| \\
& =\left|\frac{1}{\sqrt{n}} \sum_{i=1}^{n} A_{i}(s) \phi\left(X_{i}(s)\right)\left(d\left(X_{i}(s), V^{n}(s)\right)-d\left(X_{i}(s), \widetilde{V}^{n}(s)\right)\right)\right| \\
& \leq \frac{1}{\sqrt{n}} \sum_{i=1}^{n} A_{i}(s)\left|\phi\left(X_{i}(s)\right)\right| K \rho\left(V^{n}(s), \widetilde{V}^{n}(s)\right),
\end{aligned}
$$

we have

$$
\begin{aligned}
\mathbb{E} \mid & \sqrt{n}\left\langle\left.\phi\left(d\left(\cdot, V^{n}(s)\right)-d\left(\cdot, \widetilde{V}^{n}(s)\right), \widetilde{V}^{n}(s)\right\rangle\right|^{2 \lambda} 1_{\left\{\eta_{m}^{n, p} \geq s\right\}}\right. \\
\leq & \left.\left.K^{2 \lambda} n^{\lambda} \mathbb{E}\left|\frac{1}{n} \sum_{i=1}^{n} A_{i}(s) c_{7} e^{\left|X_{i}(s)\right|}\right| \widetilde{\phi}\right|_{0} \rho\left(V^{n}(s), \widetilde{V}^{n}(s)\right)\right|^{2 \lambda} 1_{\left\{\eta_{m}^{n, p} \geq s\right\}} \\
\leq & K^{2 \lambda} n^{\lambda} c_{7}^{2 \lambda}|\widetilde{\phi}|_{0}^{2 \lambda} \mathbb{E}\left[\left(\frac{1}{n} \sum_{i=1}^{n} A_{i}(s)^{2} \frac{1}{n} \sum_{i=1}^{n} e^{2\left|X_{i}(s)\right|}\right)^{\lambda}\right.
\end{aligned}
$$




$$
\begin{aligned}
& \left.\times 2^{2 \lambda-1}\left(m^{2 \lambda}\left(\frac{1}{n} \sum_{i=1}^{n}\left|X_{i}^{n}(s)-X_{i}(s)\right|^{2}\right)^{\lambda}+\left(\frac{1}{n} \sum_{i=1}^{n}\left|A_{i}^{n}(s)-A_{i}(s)\right|\right)^{2 \lambda}\right) 1_{\left\{\eta_{m}^{n, p} \geq s\right\}}\right] \\
\leq & K^{2 \lambda} n^{\lambda} c_{7}^{2 \lambda}|\widetilde{\phi}|_{0}^{2 \lambda}\left(m^{2} c(m, 2)\right)^{\lambda} 2^{2 \lambda-1} \\
& \times \mathbb{E}\left[m^{2 \lambda} \frac{1}{n} \sum_{i=1}^{n}\left|X_{i}^{n}(s)-X_{i}(s)\right|^{2 \lambda}+\left(\frac{1}{n} \sum_{i=1}^{n}\left|A_{i}^{n}(s)-A_{i}(s)\right|^{\lambda}\right)^{2} 1_{\left\{\eta_{m}^{n, p} \geq s\right\}}\right] \\
\leq & c_{10}(m, \lambda, s)|\widetilde{\phi}|_{0}^{2 \lambda} .
\end{aligned}
$$

As

$$
\begin{aligned}
\mid \sqrt{n} & \left\langle\phi\left(d\left(\cdot, \widetilde{V}^{n}(s)\right)-d(\cdot, V(s))\right), \widetilde{V}^{n}(s)\right\rangle \mid \\
\quad & \left.=\mid \frac{1}{\sqrt{n}} \sum_{i=1}^{n} A_{i}(s) \phi\left(X_{i}(s)\right)\left(d\left(X_{i}(s), \widetilde{V}^{n}(s)\right)\right)-d\left(X_{i}(s), V(s)\right)\right) \mid
\end{aligned}
$$

we have

$$
\begin{aligned}
& \mathbb{E}\left|\sqrt{n}\left\langle\phi\left(d\left(\cdot, \widetilde{V}^{n}(s)\right)-d(\cdot, V(s))\right), \widetilde{V}^{n}(s)\right\rangle\right|^{2 \lambda} 1_{\left\{\eta_{m}^{n, p} \geq s\right\}} \\
& \leq c_{7}^{2 \lambda} K^{2 \lambda} n^{\lambda}|\widetilde{\phi}|_{0}^{2 \lambda} \mathbb{E}\left[\left(\frac{1}{n} \sum_{i=1}^{n} A_{i}(s)^{\frac{2 \lambda}{2 \lambda-1}} e^{\frac{2 \lambda}{2 \lambda-1}\left|X_{i}(s)\right|}\right)^{2 \lambda-1}\right. \\
& \left.\quad \times \frac{1}{n} \sum_{i=1}^{n}\left|d\left(X_{i}(s), \widetilde{V}^{n}(s)\right)-d\left(X_{i}(s), V(s)\right)\right|^{2 \lambda} 1_{\left\{\eta_{m}^{n, p} \geq s\right\}}\right] \\
& \leq c_{11}(m, \lambda, s)|\widetilde{\phi}|_{0}^{2 \lambda},
\end{aligned}
$$

where the last inequality follows by arguments similar to the estimate for the third term on the right side of (2.8).

Let

$$
N_{n}=\sum_{i=1}^{n}\left(A_{i}(s) \phi\left(X_{i}(s)\right) d\left(X_{i}(s), V(s)\right)-\langle\phi d(\cdot, V(s)), V(s)\rangle\right), \quad n \geq 1 .
$$

Then $\left\{N_{n}: n=1,2, \cdots\right\}$ is a discrete-time $\mathbb{P}(\cdot \mid W)$-martingale with, using the notation of Burkholder [3],

$$
S_{n}(N)^{2}=\sum_{i=1}^{n}\left(A_{i}(s) \phi\left(X_{i}(s)\right) d\left(X_{i}(s), V(s)\right)-\langle\phi d(\cdot, V(s)), V(s)\rangle\right)^{2} .
$$

(Do not confuse $S_{n}(N)$ here with our process $S_{n}$.) By Theorem 3.2 in Burkholder [3], there exists a constant $C_{\lambda}$ such that

$$
\mathbb{E}\left(N_{n}^{2 \lambda} \mid W\right) \leq C_{\lambda} \mathbb{E}\left(S_{n}(N)^{2 \lambda} \mid W\right)
$$


Therefore

$$
\begin{aligned}
\mathbb{E} \mid & =\left.\left\langle\phi d(\cdot, V(s)), \widetilde{V}^{n}(s)-V(s)\right\rangle\right|^{2 \lambda} \\
& =\frac{1}{n^{\lambda}} \mathbb{E}\left(\mathbb{E}\left(N_{n}^{2 \lambda} \mid W\right)\right) \\
& \leq \frac{1}{n^{\lambda}} C_{\lambda} \mathbb{E}\left[\mathbb{E}\left[\left(\sum_{i=1}^{n}\left(A_{i}(s) \phi\left(X_{i}(s)\right) d\left(X_{i}(s), V(s)\right)-\langle\phi d(\cdot, V(s)), V(s)\rangle\right)^{2}\right)^{\lambda} \mid W\right]\right] \\
& =C_{\lambda} \mathbb{E}\left(\left|A_{1}(s) \phi\left(X_{1}(s)\right) d\left(X_{1}(s), V(s)\right)-\langle\phi d(\cdot, V(s)), V(s)\rangle\right|^{2 \lambda}\right) \\
& \leq c_{12}(m, \lambda, s)|\widetilde{\phi}|_{0}^{2 \lambda}
\end{aligned}
$$

and we have

$$
\left.\left.\mathbb{E}\left[\left|\sqrt{n}\left(\left\langle\phi d\left(\cdot, V^{n}(s)\right)\right), V^{n}(s)\right\rangle-\langle\phi d(\cdot, V(s))), V(s)\right\rangle\right)\right|^{2 \lambda} \mathbf{1}_{\left\{\eta_{m}^{n, p} \geq s\right\}}\right] \leq c_{13}(m, \lambda, s)|\widetilde{\phi}|_{1}^{2 \lambda} .
$$

Similar arguments give

$$
\mathbb{E}\left[\left(\sqrt{n}\left|\left\langle L\left(V^{n}(s)\right) \phi, V^{n}(s)\right\rangle-\langle L(V(s)) \phi, V(s)\rangle\right|\right)^{2 \lambda} \mathbf{1}_{\left\{\eta_{m}^{n, p} \geq s\right\}}\right] \leq c_{14}(m, \lambda, s)|\widetilde{\phi}|_{3}^{2 \lambda},
$$

where the estimate in terms of the higher derivatives is required because of the differential operator, and we have

$$
\mathbb{E}\left[H_{\phi}^{3, n}(s)^{\lambda} \mathbf{1}_{\left\{\eta_{m}^{n, p} \geq s\right\}}\right] \leq c_{15}(m, \lambda, s)|\widetilde{\phi}|_{3}^{2 \lambda} \leq c_{15}(m, \lambda, s)\|\phi\|_{4}^{2 \lambda} .
$$

Finally, again applying similar arguments, we can show

$$
\mathbb{E}\left[H_{\phi}^{2, n}(s)^{\lambda} \mathbf{1}_{\left\{\eta_{m}^{n, p} \geq s\right\}}\right] \leq c_{16}(m, \lambda, s)|\widetilde{\phi}|_{2}^{2 \lambda} \leq c_{16}(m, \lambda, s)\|\phi\|_{3}^{2 \lambda} .
$$

Without loss of generality, we can assume that all of the $c_{l}(m, \lambda, s)$ are nondecreasing in $s$. Applying (3.6), (3.7), and (3.8), (3.5) gives

$$
\mathbb{E}\left[\sup _{s \leq t}\left\langle\phi, S_{n}\left(s \wedge \eta_{m}^{n, p}\right)-S_{n}(0)\right\rangle^{2}\right] \leq c_{17}(m, p, t)\|\phi\|_{4}^{2}
$$

For $\kappa$ sufficiently large, the embedding $T_{\kappa-1}^{4}: \Phi_{\kappa-1} \rightarrow \Phi_{4}$ is Hilbert-Schmidt and hence, if $\left\{\phi_{k}\right\}$ is an orthonormal basis for $\Phi_{\kappa-1}, \sum_{k=1}^{\infty}\left\|\phi_{k}\right\|_{4}^{2}<\infty$. (See [17], Lemma 1 and Theorem 2, pages 33-34.) Consequently,

$$
\begin{aligned}
\mathbb{E}\left[\sup _{s \leq t}\left\|S_{n}\left(s \wedge \eta_{m}^{n, p}\right)-S_{n}(0)\right\|_{-(\kappa-1)}^{2}\right] & \leq \mathbb{E}\left[\sum_{k=1}^{\infty} \sup _{s \leq t}\left\langle\phi_{k}, S_{n}\left(s \wedge \eta_{m}^{n, p}\right)-S_{n}(0)\right\rangle^{2}\right] \\
& \leq c_{17}(m, p, t) \sum_{k=1}^{\infty}\left\|\phi_{k}\right\|_{4}^{2}<\infty .
\end{aligned}
$$

It follows that for each $t \geq 0$ and $\epsilon>0$, there exists $k_{t, \epsilon}>0$ such that

$$
\sup _{n} \mathbb{P}\left\{\sup _{s \leq t}\left\|S_{n}\left(s \wedge \eta_{m}^{n, p}\right)\right\|_{-(\kappa-1)}>k_{t, \epsilon}\right\} \leq \epsilon
$$

But $\left\{\psi \in \Phi_{-\kappa}:\|\psi\|_{-(\kappa-1)} \leq k_{t, \epsilon}\right\}$ is a compact subset of $\Phi_{-\kappa}$, so $\left\{S_{n}\left(\cdot \wedge \eta_{m}^{n, p}\right)\right\}$ satisfies the compact containment condition in $\Phi_{-\kappa}$. 
Similarly, by (3.4), for $t<t+h \leq T, h<1$,

$$
\begin{aligned}
& \mathbb{E}\left[\left\|S_{n}\left((t+h) \wedge \eta_{m}^{n, p}\right)-S_{n}\left(t \wedge \eta_{m}^{n, p}\right)\right\|_{-(\kappa-1)}^{2} \mid \mathcal{F}_{t}\right] \\
& \quad \leq \sum_{k=1}^{\infty} \mathbb{E}\left[\int_{t}^{t+h}\left(H_{\phi_{k}}^{1, n}(s)+H_{\phi_{k}}^{2, n}(s)+h H_{\phi_{k}}^{3, n}(s)\right) \mathbf{1}_{\left\{\eta_{m}^{n, p} \geq s\right\}} d s \mid \mathcal{F}_{t}\right] \\
& \quad \leq h^{(\lambda-1) / \lambda} \mathbb{E}\left[\sum_{k=1}^{\infty}\left(\int_{0}^{T}\left(H_{\phi_{k}}^{1, n}(s)+H_{\phi_{k}}^{2, n}(s)+h H_{\phi_{k}}^{3, n}(s)\right)^{\lambda} \mathbf{1}_{\left\{\eta_{m}^{n, p} \geq s\right\}} d s\right)^{1 / \lambda} \mid \mathcal{F}_{t}\right] .
\end{aligned}
$$

Then

$$
\begin{aligned}
\mathbb{E}\left[\sum_{k=1}^{\infty}\right. & \left.\left(\int_{0}^{T}\left(H_{\phi_{k}}^{1, n}(s)+H_{\phi_{k}}^{2, n}(s)+h H_{\phi_{k}}^{3, n}(s)\right)^{\lambda} \mathbf{1}_{\left\{\eta_{m}^{n, p} \geq s\right\}} d s\right)^{1 / \lambda}\right] \\
= & \mathbb{E}\left[\sum_{k=1}^{\infty}\left\|\phi_{k}\right\|_{4}^{2}\left(\int_{0}^{T}\left(\left(H_{\phi_{k}}^{1, n}(s)+H_{\phi_{k}}^{2, n}(s)+h H_{\phi_{k}}^{3, n}(s)\right) /\left\|\phi_{k}\right\|_{4}^{2}\right)^{\lambda} \mathbf{1}_{\left\{\eta_{m}^{n, p} \geq s\right\}} d s\right)^{1 / \lambda}\right] \\
\leq & \mathbb{E}\left[\sum_{k=1}^{\infty}\left\|\phi_{k}\right\|_{4}^{2}\left(\int_{0}^{T}\left(\left(H_{\phi_{k}}^{1, n}(s)+H_{\phi_{k}}^{2, n}(s)+h H_{\phi_{k}}^{3, n}(s)\right) /\left\|\phi_{k}\right\|_{4}^{2}\right)^{\lambda} \mathbf{1}_{\left\{\eta_{m}^{n, p} \geq s\right\}} d s\right)^{1 / \lambda}\right] \\
\leq & \left(\sum_{k=1}^{\infty}\left\|\phi_{k}\right\|_{4}^{2}\right)^{(\lambda-1) / \lambda} \\
& \quad \times\left(\sum_{k=1}^{\infty}\left\|\phi_{k}\right\|_{4}^{2} \int_{0}^{T} \mathbb{E}\left[\left(\left(H_{\phi_{k}}^{1, n}(s)+H_{\phi_{k}}^{2, n}(s)+h H_{\phi_{k}}^{3, n}(s)\right) /\left\|\phi_{k}\right\|_{4}^{2}\right)^{\lambda} \mathbf{1}_{\left\{\eta_{m}^{n, p} \geq s\right\}}\right] d s\right)^{1 / \lambda} \\
<\infty &
\end{aligned}
$$

Since

$$
\left\|S_{n}\left((t+h) \wedge \eta_{m}^{n, p}\right)-S_{n}\left(t \wedge \eta_{m}^{n, p}\right)\right\|_{-\kappa}^{2} \leq\left\|S_{n}\left((t+h) \wedge \eta_{m}^{n, p}\right)-S_{n}\left(t \wedge \eta_{m}^{n, p}\right)\right\|_{-(\kappa-1)}^{2},
$$

we have verified the conditions of Theorem 4.20 of [24] (Theorem 3.8.6 of [13]) with

$$
\gamma_{n}(\delta)=\delta^{(\lambda-1) / \lambda} \sum_{k=1}^{\infty}\left(\int_{0}^{T}\left(H_{\phi_{k}}^{1, n}(s)+H_{\phi_{k}}^{2, n}(s)+\delta H_{\phi_{k}}^{3, n}(s)\right)^{\lambda} \mathbf{1}_{\left\{\eta_{m}^{n, p} \geq s\right\}} d s\right)^{1 / \lambda} .
$$

Note that since the $S_{n}$ are continuous, tightness of $\left\{S_{n}\right\}$ in $D_{\Phi_{-k}}[0, \infty)$ implies tightness in $C_{\Phi_{-k}}[0, \infty)$.

The same argument gives tightness for $\left\{M^{1, n}\right\}$, and we have the following additional result.

Lemma 3.1. Under the conditions of Theorem 3.1, $\left\{M^{1, n}\right\}$ is tight in $C_{\Phi_{-\kappa}}[0, \infty)$.

\section{Characterization of the limit}

We need the following additional assumptions.

(S4) There exists $\delta>0$ such that

$$
\left|\sigma(x, \nu)^{T} z\right|^{2}-\delta \int_{U}|z \cdot \alpha(x, \nu, u)|^{2} \mu(d u) \geq 0
$$

$\forall x, z \in \mathbb{R}^{d}, \nu \in \mathcal{M}\left(\mathbb{R}^{d}\right)$. 
(S5) The coefficients $\sigma, c, d, a, b, \gamma, \alpha$, and $\beta$ are differentiable with respect to the measure in the sense that, for example, there exists a bounded, continuous function $\partial d$ on $\mathbb{R}^{d} \times \mathcal{M}\left(\mathbb{R}^{d}\right) \times \mathbb{R}^{d}$ such that for $\nu_{1}, \nu_{2} \in \mathcal{M}\left(\mathbb{R}^{d}\right)$,

$$
d\left(x, \nu_{2}\right)-d\left(x, \nu_{1}\right)=\int_{0}^{1} \int_{\mathbb{R}^{d}} \partial d\left(x,(1-r) \nu_{1}+r \nu_{2}, y\right)\left(\nu_{2}(d y)-\nu_{1}(d y)\right) d r .
$$

(S6) For $\kappa$ given by Theorem 3.1, $\phi \in \Phi_{\kappa+l+2}, \nu_{1}, \nu_{2} \in \mathcal{M}\left(\mathbb{R}^{d}\right)$, and $u \in U$,

$$
\begin{aligned}
& F_{1}\left(\nu_{1}, \nu_{2}\right) \phi \\
& \qquad \begin{array}{l}
\equiv d\left(\cdot, \nu_{2}\right) \phi+L\left(\nu_{2}\right) \phi \\
\quad+\int_{\mathbb{R}^{d}} \int_{0}^{1}\left(\phi(x) \partial d\left(x, r \nu_{2}+(1-r) \nu_{1}, \cdot\right)\right. \\
\left.\quad+\partial L\left(r \nu_{2}+(1-r) \nu_{1}, \cdot\right) \phi(x)\right) d r \nu_{1}(d x)
\end{array}
\end{aligned}
$$

and

$$
\begin{aligned}
& F_{2}\left(\nu_{1}, \nu_{2}, u\right) \phi \\
& \equiv \phi \beta\left(\cdot, \nu_{2}, u\right)+\nabla \phi^{T} \alpha\left(\cdot, \nu_{2}, u\right) \\
& \quad-\int_{\mathbb{R}^{d}} \int_{0}^{1}\left(\phi(x) \partial \beta\left(x, r \nu_{2}+(1-r) \nu_{1}, u, \cdot\right)\right. \\
& \left.\quad+\nabla \phi^{T}(x) \partial \alpha\left(x, r \nu_{2}+(1-r) \nu_{1}, u, \cdot\right)\right) d r \nu_{1}(d x)
\end{aligned}
$$

are in $\Phi_{\kappa+l}$ for $0 \leq l \leq 2$.

For $\phi \in \Phi_{\kappa+2}$, the mappings

$$
\left(\nu_{1}, \nu_{2}, v\right) \in \mathcal{M}\left(\mathbb{R}^{d}\right) \times \mathcal{M}\left(\mathbb{R}^{d}\right) \times \Phi_{-\kappa} \rightarrow\left\langle F_{1}\left(\nu_{1}, \nu_{2}\right) \phi, v\right\rangle \in \mathbb{R}
$$

and

$$
\left(\nu_{1}, \nu_{2}, v\right) \in \mathcal{M}\left(\mathbb{R}^{d}\right) \times \mathcal{M}\left(\mathbb{R}^{d}\right) \times \Phi_{-\kappa} \rightarrow\left\langle F_{2}\left(\nu_{1}, \nu_{2}, \cdot\right) \phi, v\right\rangle \in L^{2}(U, \mu)
$$

are continuous.

(S7) For each $\nu \in \mathcal{M}\left(\mathbb{R}^{d}\right)$, the mappings from $x \in \mathbb{R}^{d}$ to $a_{i j}(x, \nu), b_{i}(x, \nu), d(x, \nu) \in$ $\mathbb{R}$ and $\alpha_{i}(x, \nu, \cdot), \beta(x, \nu, \cdot) \in L^{2}(U, \mu)$ have bounded derivatives with respect to $x$ up to order $q \equiv \kappa+2$. For each $x \in \mathbb{R}^{d}, u \in U$ and $\nu \in \mathcal{M}\left(\mathbb{R}^{d}\right)$, $\partial a_{i j}(x, \nu, \cdot), \partial b_{i}(x, \nu, \cdot), \partial d(x, \nu, \cdot), \partial \alpha_{i}(x, \nu, u, \cdot), \partial \beta(x, \nu, u, \cdot)$ are in $\Phi_{q}$, and there exists a constant $K$ such that

$$
\begin{aligned}
\sum_{i, j} & \left\|\partial a_{i j}(x, \nu, \cdot)\right\|_{q}^{2}+\sum_{i}\left\|\partial b_{i}(x, \nu, \cdot)\right\|_{q}^{2}+\|\partial d(x, \nu, \cdot)\|_{q}^{2} \\
& +\int_{U}\left(\sum_{i}\left\|\partial \alpha_{i}(x, \nu, u, \cdot)\right\|_{q}^{2}+\|\partial \beta(x, \nu, u, \cdot)\|_{q}^{2}\right) \mu(d u) \leq K .
\end{aligned}
$$

REMARK 4.1. If $\nu_{1}=\nu_{2}=\nu$, we write $F_{i}(\nu)$ rather than $F_{i}(\nu, \nu)$. Condition (S6) implies smoothness and growth conditions on the coefficients of the differential operators. Continuity of the mapping

$$
\left(\nu_{1}, \nu_{2}\right) \in \mathcal{M}\left(\mathbb{R}^{d}\right) \times \mathcal{M}\left(\mathbb{R}^{d}\right) \rightarrow F_{1}\left(\nu_{1}, \nu_{2}\right) \phi \in \Phi_{k}
$$


would imply (4.3).

Continuing to restrict the calculations to dimension $d=1$, by $(3.3)$,

$$
\begin{aligned}
\left\langle\phi, S_{n}(t)\right\rangle= & \left\langle\phi, S_{n}(0)\right\rangle+\left\langle\phi, M^{1, n}(t)\right\rangle+\int_{0}^{t}\left\langle F_{1}\left(V(s), V^{n}(s)\right) \phi, S_{n}(s)\right\rangle d s \\
& +\int_{U \times[0, t]}\left\langle F_{2}\left(V(s), V^{n}(s), u\right) \phi, S_{n}(s)\right\rangle W(d u d s),
\end{aligned}
$$

where

$$
\begin{gathered}
M_{\phi}^{1, n}(t)=\left\langle\phi, M^{1, n}(t)\right\rangle \\
=n^{-1 / 2} \sum_{i=1}^{n} \int_{0}^{t} A_{i}^{n}(s)\left(\phi\left(X_{i}^{n}(s)\right) \gamma\left(X_{i}^{n}(s), V^{n}(s)\right)\right. \\
\left.+\phi^{\prime}\left(X_{i}^{n}(s)\right) \sigma\left(X_{i}^{n}(s), V^{n}(s)\right)\right) d B_{i}(s), \\
\sqrt{n}\left(\left\langle\phi d\left(\cdot, V^{n}(s)\right)+L\left(V^{n}(s)\right) \phi, V^{n}(s)\right\rangle-\langle\phi d(\cdot, V(s))+L(V(s)) \phi, V(s)\rangle\right) \\
=\left\langle\phi d\left(\cdot, V^{n}(s)\right)+L\left(V^{n}(s)\right) \phi, S_{n}(s)\right\rangle \\
\quad+\sqrt{n}\left\langle\phi d\left(\cdot, V^{n}(s)\right)+L\left(V^{n}(s)\right) \phi-\phi d(\cdot, V(s))+L(V(s)) \phi, V(s)\right\rangle \\
=\left\langle\phi d\left(\cdot, V^{n}(s)\right)+L\left(V^{n}(s)\right) \phi, S_{n}(s)\right\rangle \\
\quad+\int_{\mathbb{R}} \int_{0}^{1}\left\langle\phi(x) \partial d\left(x, r V^{n}(s)+(1-r) V(s), \cdot\right)\right. \\
\left.\quad+\partial L\left(r V^{n}(s)+(1-r) V(s), \cdot\right) \phi(x), S_{n}(s)\right\rangle d r V(s, d x) \\
=\left\langle F_{1}\left(V(s), V^{n}(s)\right) \phi, S_{n}(s)\right\rangle,
\end{gathered}
$$

and

$$
\begin{gathered}
\sqrt{n}\left(\left\langle\phi \beta\left(\cdot, V^{n}(s), u\right)+\phi^{\prime} \alpha\left(\cdot, V^{n}(s), u\right), V^{n}(s)\right\rangle-\left\langle\phi \beta(\cdot, V(s), u)+\phi^{\prime} \alpha(\cdot, V(s), u), V(s)\right\rangle\right) \\
=\left\langle\phi \beta\left(\cdot, V^{n}(s), u\right)+\phi^{\prime} \alpha\left(\cdot, V^{n}(s), u\right), S_{n}(s)\right\rangle \\
-\int_{\mathbb{R}} \int_{0}^{1}\left\langle\phi(x) \partial \beta\left(x, r V^{n}(s)+(1-r) V(s), u, \cdot\right)\right. \\
\left.\quad+\phi^{\prime}(x) \partial \alpha(x, V(s), u, \cdot), S_{n}(s)\right\rangle d r V(s, d x) \\
=\left\langle F_{2}\left(V(s), V^{n}(s), u\right) \phi, S_{n}(s)\right\rangle .
\end{gathered}
$$

Let $\mathbb{H}=L^{2}(U, \mu)$. In the terminology of Kurtz and Protter [25], we define a $\mathbb{R} \times \mathbb{H}^{\#}$-semimartingale $Y$ by setting

$$
Y(a, h, t)=a t+B^{h}(t)=a t+\int_{U \times[0, t]} h(u) W(d u d s),
$$

for $a \in \mathbb{R}$ and $h \in \mathbb{H}$. Let

$$
U_{n}(t)=S_{n}(0)+M^{1, n}(t),
$$

and for $\phi \in \Phi_{\kappa+2}$, let $\left\langle F\left(V(s), V^{n}(s)\right) \phi, S_{n}(s)\right\rangle$ denote the $\mathbb{R} \times \mathbb{H}$-valued process given by

$$
\left\langle F\left(V(s), V^{n}(s)\right) \phi, S_{n}(s)\right\rangle=\left(\left\langle F_{1}\left(V(s), V^{n}(s)\right) \phi, S_{n}(s)\right\rangle,\left\langle F_{2}\left(V(s), V^{n}(s), u\right) \phi, S_{n}(s)\right\rangle\right) .
$$


Then (4.5) can be rewritten in the notation of Kurtz and Protter [25] as

$$
\left\langle\phi, S_{n}(t)\right\rangle=\left\langle\phi, U_{n}(t)\right\rangle+\left\langle F\left(V(\cdot), V^{n}(\cdot)\right) \phi, S_{n}\right\rangle \cdot Y(t) .
$$

Note that for each $\phi \in C^{1}(\mathbb{R}), h \in \mathbb{H}$, and $1 \leq i \leq n,\left[M_{\phi}^{1, n}, B^{h}\right]_{t}=0$,

$$
\begin{aligned}
& {\left[M_{\phi}^{1, n}, B_{i}\right]_{t} } \\
= & \frac{1}{n} \int_{0}^{t}\left(A_{i}^{n}(s)\right)^{2}\left(\phi\left(X_{i}^{n}(s)\right) \gamma\left(X_{i}^{n}(s), V^{n}(s)\right)+\phi^{\prime}\left(X_{i}^{n}(s)\right) \sigma\left(X_{i}^{n}(s), V^{n}(s)\right)\right)^{2} d s,
\end{aligned}
$$

and

$$
\left[M_{\phi}^{1, n}\right]_{t}=\int_{0}^{t}\left\langle\left(\phi \gamma\left(\cdot, V^{n}(s)\right)+\phi^{\prime} \sigma\left(\cdot, V^{n}(s)\right)\right)^{2}, V_{2}^{n}(s)\right\rangle d s
$$

where $V_{2}^{n}(s)=\frac{1}{n} \sum_{j=1}^{n} A_{j}^{n}(s)^{2} \delta_{X_{j}^{n}(s)}$.

We should emphasize that we are proving convergence in distribution for $\left\{S_{n}\right\}$. The limit will not "live" on the original probability space. To be precise, for a countable dense subset $\left\{h_{j}\right\} \subset \mathbb{H}$, the sequence

$$
\left\{\left(V^{n}, M^{1, n}, S_{n},\left\{Y\left(h_{j}\right)\right\},\left\{B_{i}\right\},\left\{X_{i}\right\},\left\{A_{i}\right\}\right)\right\}
$$

is relatively compact in $C_{\mathcal{M}(\mathbb{R}) \times\left(\Phi_{-\kappa}\right)^{2} \times\left(\mathbb{R}^{\infty}\right)^{4}}[0, \infty)$. Denoting a limit point by

$$
\left(V, M, S,\left\{Y\left(h_{i}\right)\right\},\left\{B_{i}\right\},\left\{X_{i}\right\},\left\{A_{i}\right\}\right)
$$

(even though these are not the $V, Y,\left\{B_{i}\right\},\left\{X_{i}\right\},\left\{A_{i}\right\}$ on the original probability space, they will have the same distribution), $M$ (and hence $S$ ) will not be adapted to the filtration $\left\{\mathcal{F}_{t}^{Y,\left\{B_{i}\right\}}\right\}$ generated by $Y$ and $\left\{B_{i}\right\}$. Note that $\left\{Y\left(h_{i}\right)\right\}$ determines $Y(h)$ (and hence $B^{h}$ ) for all $h \in \mathbb{H}$ and the $Y(h)$ determine $W$.

For any limit point, $M$ will be a $\Phi_{-\kappa}$-valued local martingale with $\left[M_{\phi}, B^{h}\right]_{t}=0$ for every $\phi \in \Phi$ and $h \in \mathbb{H}$ and

$$
\left[M_{\phi}\right]_{t}=\int_{0}^{t}\left\langle\left(\phi \gamma(\cdot, V(s))+\phi^{\prime} \sigma(\cdot, V(s))\right)^{2}, V_{2}(s)\right\rangle d s
$$

where

$$
V_{2}(t)=\lim _{n \rightarrow \infty} \frac{1}{n} \sum_{i=1}^{n} A_{i}(t)^{2} \delta_{X_{i}(t)}
$$

LEMma 4.1. For $\phi \in \Phi_{\kappa}$,

$$
\begin{aligned}
\mathbb{E} & {\left[e^{i\langle\phi, S(0)+M(t)\rangle} \mid W\right] } \\
& =\exp \left\{-\frac{1}{2}\left(\left\langle\phi^{2}, V_{2}(0)\right\rangle-\langle\phi, V(0)\rangle^{2}+\int_{0}^{t}\left\langle\left(\phi \gamma(\cdot, V(s))+\phi^{\prime} \sigma(\cdot, V(s))\right)^{2}, V_{2}(s)\right\rangle d s\right)\right\}
\end{aligned}
$$

and

$$
\begin{aligned}
\mathbb{E} & {\left[e^{i\langle\phi, M(t+r)-M(t)\rangle} \mid \sigma(W) \vee \mathcal{F}_{t}^{M}\right] } \\
& \left.=\exp \left\{-\frac{1}{2} \int_{t}^{t+r}\left\langle\left(\phi \gamma(\cdot, V(s))+\phi^{\prime} \sigma(\cdot, V(s))\right)^{2}, V_{2}(s)\right\rangle d s\right)\right\},
\end{aligned}
$$


which determine the joint distribution of $W$ and $M$.

Proof: Define

$$
\begin{aligned}
& \widetilde{M}_{\phi}^{n}(t)=\left\langle\phi, \widetilde{M}^{n}(t)\right\rangle \\
&=n^{-1 / 2} \sum_{i=1}^{n} \int_{0}^{t} A_{i}(s)\left(\phi\left(X_{i}(s)\right) \gamma\left(X_{i}(s), V(s)\right)\right. \\
&\left.\quad+\phi^{\prime}\left(X_{i}(s)\right) \sigma\left(X_{i}(s), V(s)\right)\right) d B_{i}(s),
\end{aligned}
$$

and observe that

$$
\begin{aligned}
& {\left[M_{\phi}^{1, n}-\widetilde{M}_{\phi}^{n}\right]_{t}} \\
& \quad=\frac{1}{n} \sum_{i=1}^{n} \int_{0}^{t}\left(A_{i}^{n}(s) \phi\left(X_{i}^{n}(s)\right) \gamma\left(X_{i}^{n}(s), V^{n}(s)\right)+\phi^{\prime}\left(X_{i}^{n}(s)\right) \sigma\left(X_{i}^{n}(s), V^{n}(s)\right)\right. \\
& \left.\quad-A_{i}(s) \phi\left(X_{i}(s)\right) \gamma\left(X_{i}(s), V(s)\right)-\phi^{\prime}\left(X_{i}(s)\right) \sigma\left(X_{i}(s), V(s)\right)\right)^{2} d s .
\end{aligned}
$$

converges to zero. It follows that $M^{1, n}$ and $\widetilde{M}^{n}$ must have the same limit. Again, to be precise, one should say that any limit point of

$$
\left\{\left(V^{n}, M^{1, n}, \widetilde{M}^{n}, S_{n},\left\{Y\left(h_{j}\right)\right\},\left\{B_{i}\right\},\left\{X_{i}\right\},\left\{A_{i}\right\}\right)\right\}
$$

will be of the form

$$
\left(V, M, M, S,\left\{Y\left(h_{i}\right)\right\},\left\{B_{i}\right\},\left\{X_{i}\right\},\left\{A_{i}\right\}\right) .
$$

For a $\sigma(W)$ measurable random variable $Z$, exchangeability implies

$$
\begin{aligned}
\mathbb{E} & {\left[e^{i\langle\phi, S(0)+M(t)\rangle} Z\right] } \\
& =\lim _{n \rightarrow \infty} \mathbb{E}\left[e^{i\left\langle\phi, S_{n}(0)+\widetilde{M}^{n}(t)\right\rangle} Z\right] \\
& =\lim _{n \rightarrow \infty} \mathbb{E}\left[\mathbb{E}\left[\exp \left\{i \frac{1}{\sqrt{n}}\left(A_{1}(0) \phi\left(X_{1}(0)\right)-\langle\phi, V(0)\rangle+\widetilde{M}_{\phi, 1}\right\} \mid W\right]^{n} Z\right]\right. \\
& =\mathbb{E}\left[\exp \left\{-\frac{1}{2}\left(\left\langle\phi^{2}, V_{2}(0)\right\rangle-\langle\phi, V(0)\rangle^{2}+\int_{0}^{t}\left\langle\left(\phi \gamma(\cdot, V(s))+\phi^{\prime} \sigma(\cdot, V(s))\right)^{2}, V_{2}(s)\right\rangle d s\right)\right\} Z\right]
\end{aligned}
$$

where

$$
\widetilde{M}_{\phi, 1}(t)=\int_{0}^{t} A_{1}(s)\left(\phi\left(X_{1}(s)\right) \gamma\left(X_{1}(s), V(s)\right)+\phi^{\prime}\left(X_{1}(s)\right) \sigma\left(X_{1}(s), V(s)\right)\right) d B_{1}(s) .
$$

The proof of the second identity is similar.

Let $U(t)=S(0)+M(t)$ and

$$
\langle F(V(s)) \phi, S(s)\rangle=\left(\left\langle F_{1}(V(s)) \phi, S(s)\right\rangle,\left\langle F_{2}(V(s), u) \phi, S(s)\right\rangle\right) .
$$

Then $\left(S_{n}, U_{n}\right)$ is relatively compact in $C_{\Phi_{-\kappa} \times \Phi_{-\kappa}}[0, \infty)$, and by the continuity assumptions on $F_{1}$ and $F_{2}$ and Theorem 5.5 in [25], for any limit point $(S, U)$, we have

$$
\langle\phi, S(t)\rangle=\langle\phi, U(t)\rangle+\langle F(V(\cdot)) \phi, S\rangle \cdot Y(t) .
$$


Specifically, any limit point of $\left\{S_{n}\right\}$ satisfies (1.10).

To prove uniqueness for the solution to (1.10), suppose that $S_{1}$ and $S_{2}$ are solutions and set $\xi=S_{1}-S_{2}$. Then $\xi$ satisfies

$$
\langle\phi, \xi(t)\rangle=\int_{0}^{t}\left\langle F_{1}(V(s)) \phi, \xi(s)\right\rangle d s+\int_{U \times[0, t]}\left\langle F_{2}(V(s), u) \phi, \xi(s)\right\rangle W(d u d s) .
$$

We adapt arguments of Rozovskii [36] to establish that $\xi \equiv 0$ is the unique solution to (4.6) and hence establish uniqueness for (1.10).

Lemma 4.2. Suppose that the assumptions (S1)-(S7) hold. Then $\xi=0$ a.s.

Proof: Let $q=\kappa+2$, and for $\nu \in \mathcal{M}(\mathbb{R})$, define $\rho_{\nu} \equiv \int_{\mathbb{R}} \psi(x)^{-1} \nu(d x)<\infty$. By LemmaA.6,

$$
2\left\langle v, F_{1}^{*}(\nu) v\right\rangle_{-q}+\int_{U}\left\|F_{2}^{*}(\nu, u) v\right\|_{-q}^{2} \mu(d u) \leq c_{18} \rho_{\nu}^{2}\|v\|_{-q}^{2},
$$

for all $v \in \Phi_{-\kappa}$.

Note that $\xi(t)$ takes values in $\Phi_{-\kappa} \subset \Phi_{-q}$. Let $\left\{\phi_{j}^{q}\right\}$ be an orthonormal basis for $\Phi_{q}$. Applying Itô's formula, we have

$$
\begin{aligned}
\left\langle\phi_{j}^{q}, \xi(t)\right\rangle^{2}=\int_{0}^{t} 2\left\langle\phi_{j}^{q},\right. & \xi(t)\rangle\left\langle F_{1}(V(s)) \phi_{j}^{q}, \xi(s)\right\rangle d s \\
& +\int_{U \times[0, t]} 2\left\langle\phi_{j}^{q}, \xi(t)\right\rangle\left\langle F_{2}(V(s), u) \phi_{j}^{q}, \xi(s)\right\rangle W(d u d s) \\
& +\int_{0}^{t} \int_{U}\left\langle F_{2}(V(s), u) \phi_{j}^{q}, \xi(s)\right\rangle^{2} \mu(d u) d s .
\end{aligned}
$$

By Proposition 2.1, if (2.1) and (2.3) hold, then (2.6) holds giving $\mathbb{E}\left[\sup _{t \leq T} \rho_{V(t)}\right]<\infty$. Let $\tau_{k}=\inf \left\{t: \rho_{V(t)} \geq k\right\}$. Stopping the processes at $\tau_{k}$, taking expectations, and summing over $j,(4.7)$ gives

$$
\begin{aligned}
\mathbb{E}\left\|\xi\left(t \wedge \tau_{k}\right)\right\|_{-q}^{2} & =\mathbb{E} \int_{0}^{t \wedge \tau_{k}}\left(2\left\langle\xi(s), F_{1}^{*}(V(s)) \xi(s)\right\rangle_{-q}+\int_{U}\left\|F_{2}^{*}(V(s), u) \xi(s)\right\|_{-q}^{2} \mu(d u)\right) d s \\
& \leq \int_{0}^{t} c_{18} k^{2} \mathbb{E}\left\|\xi\left(s \wedge \tau_{k}\right)\right\|_{-q}^{2} d s .
\end{aligned}
$$

Then uniqueness follows from Gronwall's inequality and the fact that $\tau_{k} \rightarrow \infty$ as $k \rightarrow$ $\infty$

Finally, we have our main result.

THEOREM 4.2. Under assumptions (S1)-(S7), we have $S_{n} \Rightarrow S$ and $S$ is the unique solution to the stochastic evolution equation (1.10).

\section{CLT for Euler scheme}

Now we consider the CLT for the Euler scheme used in [27]. Let $\eta_{\delta}(s)=\left[\frac{s}{\delta}\right] \delta$, and for some partition $\left\{U_{k}\right\}$ of $U$ and $u_{k} \in U_{k}$, define $\xi_{\delta}(u)=u_{k}, u \in U_{k}, k=1,2, \ldots$. 
Let $\left\{\left(X_{i}^{n, \delta}, A_{i}^{n, \delta}\right), i=1, \ldots, n\right\}$ satisfy

$$
\begin{aligned}
X_{i}^{n, \delta}(t)= & X_{i}(0)+\int_{0}^{t} \sigma\left(X_{i}^{n, \delta}\left(\eta_{\delta}(s)\right), V^{n, \delta}\left(\eta_{\delta}(s)\right)\right) d B_{i}(s) \\
& +\int_{0}^{t} c\left(X_{i}^{n, \delta}\left(\eta_{\delta}(s)\right), V^{n, \delta}\left(\eta_{\delta}(s)\right)\right) d s \\
& +\int_{U \times[0, t]} \alpha\left(X_{i}^{n, \delta}\left(\eta_{\delta}(s)\right), V^{n, \delta}\left(\eta_{\delta}(s)\right), \xi_{\delta}(u)\right) W(d u d s),
\end{aligned}
$$

$$
\begin{aligned}
A_{i}^{n, \delta}(t)= & A_{i}(0)+\int_{0}^{t} A_{i}^{n, \delta}(s) \gamma\left(X_{i}^{n, \delta}\left(\eta_{\delta}(s)\right), V^{n, \delta}\left(\eta_{\delta}(s)\right)\right) d B_{i}(s) \\
& +\int_{0}^{t} A_{i}^{n, \delta}(s) d\left(X_{i}^{n, \delta}\left(\eta_{\delta}(s)\right), V^{n, \delta}\left(\eta_{\delta}(s)\right)\right) d s \\
& +\int_{U \times[0, t]} A_{i}^{n, \delta}(s) \beta\left(X_{i}^{n, \delta}\left(\eta_{\delta}(s)\right), V^{n, \delta}\left(\eta_{\delta}(s)\right), \xi_{\delta}(u)\right) W(d u d s),
\end{aligned}
$$

where

$$
V^{n, \delta}(t)=\frac{1}{n} \sum_{i=1}^{n} A_{i}^{n, \delta}(t) \delta_{X_{i}^{n, \delta}(t)} .
$$

In this paper, we only analyze the simplest case in which $W$ is a one-dimensional Brownian motion, that is, $U$ consists of a single point.

Modifying Theorem 3.3 in [27] in a way similar to the proof of Theorem 2.4 of the current paper, we have the following result.

THEOREM 5.1. Under the assumptions (S1)-(S5), we have

$$
\mathbb{E} \sup _{0 \leq t \leq T}\left(\left|X_{i}^{n, \delta}(t)-X_{i}^{n}(t)\right|^{2 \lambda}+\left(\frac{1}{n} \sum_{j=1}^{n}\left|A_{j}^{n, \delta}(t)-A_{j}^{n}(t)\right|^{\lambda}\right)^{2}\right) 1_{t<\eta_{m}^{n, \delta}} \leq c(T, m) \delta^{\lambda},
$$

where

$$
\eta_{m}^{n, \delta}=\inf \left\{t: \frac{1}{n} \sum_{i=1}^{n} A_{i}^{n}(t)^{2}>m^{2} \text { or } \frac{1}{n} \sum_{i=1}^{n} A_{i}^{n, \delta}(t)^{2}>m^{2}\right\} .
$$

Applying the same arguments as those in Section 3, we can prove that the sequence $\widetilde{S}_{n} \equiv \sqrt{n}\left(V^{n, 1 / n}-V^{n}\right)$ is tight. Now we characterize its limit points. 
Note that $\widetilde{S}_{n}(0)=0$. As in (3.3), we have

$$
\begin{aligned}
& \left\langle\phi, \widetilde{S}_{n}(t)\right\rangle \\
& =\frac{1}{\sqrt{n}} \sum_{i=1}^{n} \int_{0}^{t}\left\{A _ { i } ^ { n , 1 / n } ( s ) \left[\phi\left(X_{i}^{n, 1 / n}(s)\right) \gamma\left(X_{i}^{n, 1 / n}\left(\eta_{\frac{1}{n}}(s)\right), V^{n, 1 / n}\left(\eta_{\frac{1}{n}}(s)\right)\right)\right.\right. \\
& \left.+\phi^{\prime}\left(X_{i}^{n, 1 / n}(s)\right) \sigma\left(X_{i}^{n, 1 / n}\left(\eta_{\frac{1}{n}}(s)\right), V^{n, 1 / n}\left(\eta_{\frac{1}{n}}(s)\right)\right)\right] \\
& \left.\quad-A_{i}^{n}(s)\left[\phi\left(X_{i}^{n}(s)\right) \gamma\left(X_{i}^{n}(s), V^{n}(s)\right)+\phi^{\prime}\left(X_{i}^{n}(s)\right) \sigma\left(X_{i}^{n}(s), V^{n}(s)\right)\right]\right\} d B_{i}(s) \\
& +\frac{1}{\sqrt{n}} \sum_{i=1}^{n} \int_{0}^{t}\left\{A _ { i } ^ { n , 1 / n } ( s ) \left[\phi\left(X_{i}^{n, 1 / n}(s)\right) d\left(X_{i}^{n, 1 / n}\left(\eta_{\frac{1}{n}}(s)\right), V^{n, 1 / n}\left(\eta_{\frac{1}{n}}(s)\right)\right)\right.\right. \\
& \quad+L\left(V^{n, 1 / n}\left(\eta_{\frac{1}{n}}(s)\right)\right) \phi\left(X_{i}^{n, 1 / n}\left(\eta_{\frac{1}{n}}(s)\right)\right] \\
& \left.\quad-A_{i}^{n}(s)\left[\phi\left(X_{i}^{n}(s)\right) d\left(X_{i}^{n}(s), V^{n}(s)\right)+L\left(V^{n}(s)\right) \phi\left(X_{i}^{n}(s)\right)\right]\right\} d s \\
& +\frac{1}{\sqrt{n}} \sum_{i=1}^{n} \int_{0}^{t}\left\{A _ { i } ^ { n , 1 / n } ( s ) \left[\phi\left(X_{i}^{n, 1 / n}(s)\right) \beta\left(X_{i}^{n, 1 / n}\left(\eta_{\frac{1}{n}}(s)\right), V^{n, 1 / n}\left(\eta_{\frac{1}{n}}(s)\right)\right)\right.\right. \\
& \left.+\phi^{\prime}\left(X_{i}^{n, 1 / n}(s)\right) \alpha\left(X_{i}^{n, 1 / n}\left(\eta_{\frac{1}{n}}(s)\right), V^{n, 1 / n}\left(\eta_{\frac{1}{n}}(s)\right)\right)\right] \\
& \left.\quad-A_{i}^{n}(s)\left[\phi\left(X_{i}^{n}(s)\right) \beta\left(X_{i}^{n}(s), V^{n}(s)\right)+\phi^{\prime}\left(X_{i}^{n}(s)\right) \alpha\left(X_{i}^{n}(s), V^{n}(s)\right)\right]\right\} d W(s) \\
&
\end{aligned}
$$

LEMMA 5.1. Let $\xi_{i}^{n}(t)$ be processes satisfying

$$
\frac{1}{n} \sum_{i=1}^{n} \xi_{i}^{n}(t)^{2} \leq K, \quad \forall t, \forall \omega .
$$

Then

$$
\begin{gathered}
\frac{1}{\sqrt{n}} \sum_{i=1}^{n} \int_{0}^{t} \xi_{i}^{n}(s)\left(B_{i}(s)-B_{i}\left(\eta_{\frac{1}{n}}(s)\right)\right)^{2} d s \rightarrow 0 \\
\frac{1}{\sqrt{n}} \sum_{i=1}^{n} \int_{0}^{t} \xi_{i}^{n}(s)\left(s-\eta_{\frac{1}{n}}(s)\right)^{2} d s \rightarrow 0, \\
\frac{1}{\sqrt{n}} \sum_{i=1}^{n} \int_{0}^{t} \xi_{i}^{n}(s)\left(W(s)-W\left(\eta_{\frac{1}{n}}(s)\right)\right)^{2} d s \rightarrow 0, \\
\frac{1}{\sqrt{n}} \sum_{i=1}^{n} \int_{0}^{t} \xi_{i}^{n}\left(\eta_{\frac{1}{n}}(s)\right)\left(B_{i}(s)-B_{i}\left(\eta_{\frac{1}{n}}(s)\right)\right) d s \rightarrow 0
\end{gathered}
$$




$$
\begin{gathered}
\frac{1}{\sqrt{n}} \sum_{i=1}^{n} \int_{0}^{t} \xi_{i}^{n}\left(\eta_{\frac{1}{n}}(s)\right)\left(s-\eta_{\frac{1}{n}}(s)\right) d s \rightarrow 0, \\
\frac{1}{\sqrt{n}} \sum_{i=1}^{n} \int_{0}^{t} \xi_{i}^{n}\left(\eta_{\frac{1}{n}}(s)\right)\left(W(s)-W\left(\eta_{\frac{1}{n}}(s)\right)\right) d s \rightarrow 0, \\
\frac{1}{\sqrt{n}} \sum_{i=1}^{n} \int_{0}^{t} \xi_{i}^{n}\left(\eta_{\frac{1}{n}}(s)\right)\left(B_{i}(s)-B_{i}\left(\eta_{\frac{1}{n}}(s)\right)\right) d W(s) \rightarrow 0, \\
\frac{1}{\sqrt{n}} \sum_{i=1}^{n} \int_{0}^{t} \xi_{i}^{n}\left(\eta_{\frac{1}{n}}(s)\right)\left(s-\eta_{\frac{1}{n}}(s)\right) d W(s) \rightarrow 0 .
\end{gathered}
$$

Proof: Note that

$$
\begin{aligned}
& \mathbb{E}\left|\frac{1}{\sqrt{n}} \sum_{i=1}^{n} \int_{0}^{t} \xi_{i}^{n}(s)\left(B_{i}(s)-B_{i}\left(\eta_{\frac{1}{n}}(s)\right)\right)^{2} d s\right|^{2} \\
\leq & n \mathbb{E} \int_{0}^{t} \frac{1}{n} \sum_{i=1}^{n} \xi_{i}^{n}(s)^{2} \frac{1}{n} \sum_{i=1}^{n}\left(B_{i}(s)-B_{i}\left(\eta_{\frac{1}{n}}(s)\right)\right)^{4} d s \\
\leq & K \sum_{i=1}^{n} \int_{0}^{t} 3\left(s-\eta_{\frac{1}{n}}(s)\right)^{2} d s \\
\leq & 3 K n \sum_{j=0}^{[n t]} \int_{\frac{j}{n}}^{\frac{j+1}{n}}\left(s-\frac{j}{n}\right)^{2} d s \\
= & K n[n t] \frac{1}{n^{3}} \rightarrow 0 .
\end{aligned}
$$

This proves (5.5). (5.6), (5.7) and (5.9) can be proved similarly.

For $k=1,2, \cdots$, let

$$
M_{k}=\frac{1}{\sqrt{n}} \sum_{i=1}^{n} \int_{0}^{\frac{k}{n}} \xi_{i}^{n}\left(\eta_{\frac{1}{n}}(s)\right)\left(B_{i}(s)-B_{i}\left(\eta_{\frac{1}{n}}(s)\right)\right) d s .
$$

Then $M_{k}$ is a discrete-time, square integrable martingale with quadratic variation process

$$
[M]_{k}=\sum_{j=1}^{k}\left(\frac{1}{\sqrt{n}} \sum_{i=1}^{n} \xi_{i}^{n}\left(\eta_{\frac{1}{n}}(s)\right) \int_{\frac{j-1}{n}}^{\frac{j}{n}}\left(B_{i}(s)-B_{i}\left(\eta_{\frac{1}{n}}(s)\right)\right) d s\right)^{2}
$$


Hence

$$
\begin{aligned}
\mathbb{E} M_{[n t]}^{2} & =\sum_{j=1}^{[n t]} \mathbb{E}\left(\int_{\frac{j-1}{n}}^{\frac{j}{n}} \frac{1}{\sqrt{n}} \sum_{i=1}^{n} \xi_{i}^{n}\left(\frac{j-1}{n}\right)\left(B_{i}(s)-B_{i}\left(\frac{j-1}{n}\right)\right) d s\right)^{2} \\
& =\sum_{j=1}^{[n t]} \int_{\frac{j-1}{n}}^{\frac{j}{n}} \int_{\frac{j-1}{n}}^{\frac{j}{n}} \frac{1}{n} \sum_{i=1}^{n} \mathbb{E}\left(\xi_{i}^{n}\left(\frac{j-1}{n}\right)^{2}\right)\left(s_{1} \wedge s_{2}-\frac{j-1}{n}\right) d s_{1} d s_{2} \\
& \leq K \sum_{j=1}^{[n t]} \int_{0}^{\frac{1}{n}} \int_{0}^{\frac{1}{n}} s_{1} \wedge s_{2} d s_{1} d s_{2} \\
& =2 K[n t] \int_{0}^{\frac{1}{n}} s_{1}\left(\frac{1}{n}-s_{1}\right) d s_{1} \\
& =\frac{K[n t]}{3 n^{3}} \rightarrow 0 .
\end{aligned}
$$

This proves (5.8). (5.10) can be proved similarly.

Finally,

$$
\begin{aligned}
& \mathbb{E}\left|\frac{1}{\sqrt{n}} \sum_{i=1}^{n} \int_{0}^{t} \xi_{i}^{n}\left(\eta_{\frac{1}{n}}(s)\right)\left(B_{i}(s)-B_{i}\left(\eta_{\frac{1}{n}}(s)\right)\right) d W(s)\right|^{2} \\
= & \mathbb{E} \int_{0}^{t}\left(\frac{1}{\sqrt{n}} \sum_{i=1}^{n} \xi_{i}^{n}\left(\eta_{\frac{1}{n}}(s)\right)\left(B_{i}(s)-B_{i}\left(\eta_{\frac{1}{n}}(s)\right)\right)\right)^{2} d s \\
= & \int_{0}^{t} \frac{1}{n} \sum_{i=1}^{n} \mathbb{E} \xi_{i}^{n}\left(\eta_{\frac{1}{n}}(s)\right)^{2}\left(s-\eta_{\frac{1}{n}}(s)\right) d s \\
\leq & K \sum_{j=0}^{[n t]} \int_{\frac{j}{n}}^{\frac{j+1}{n}}\left(s-\frac{j}{n}\right) d s \\
= & K([n t]+1) \frac{1}{2 n^{2}} \rightarrow 0
\end{aligned}
$$

which proves (5.11). (5.12) can be proved similarly.

LEMMA 5.2. Let

$$
\widetilde{W}^{n}(t)=\int_{0}^{t} \sqrt{2 n}\left(W(s)-W\left(\eta_{\frac{1}{n}}(s)\right)\right) d W(s) .
$$

Then $\widetilde{W} n \widetilde{W}$ and $\widetilde{W}$ is a one-dimensional Brownian motion independent of $W$. Proof: It is clear that $\widetilde{W}^{n}$ is a sequence of martingales and

$$
\begin{aligned}
{\left[\widetilde{W}^{n}\right]_{t} } & =\int_{0}^{t} 2 n\left(W(s)-W\left(\eta_{\frac{1}{n}}(s)\right)\right)^{2} d s \\
& =\frac{1}{n t} \sum_{j=1}^{n t}\left[2 n^{2} t \int_{\frac{j-1}{n}}^{\frac{j}{n}}\left(W(s)-W\left(\frac{j-1}{n}\right)\right)^{2} d s\right] \\
& \rightarrow 2 n^{2} t \int_{0}^{\frac{1}{n}} s d s=t .
\end{aligned}
$$


By (5.10), we have

$$
\left[\widetilde{W}^{n}, W\right]_{t}=\int_{0}^{t} \sqrt{n}\left(W(s)-W\left(\eta_{\frac{1}{n}}(s)\right)\right) d s \rightarrow 0,
$$

and the lemma follows by the martingale central limit theorem

Note that

$$
\begin{aligned}
& X_{i}^{n, 1 / n}(s)-X_{i}^{n, 1 / n}\left(\eta_{\frac{1}{n}}(s)\right) \\
= & \sigma\left(X_{i}^{n, 1 / n}\left(\eta_{\frac{1}{n}}(s)\right), V^{n, 1 / n}\left(\eta_{\frac{1}{n}}(s)\right)\right)\left(B_{i}(s)-B_{i}\left(\eta_{\frac{1}{n}}(s)\right)\right) \\
& +c\left(X_{i}^{n, 1 / n}\left(\eta_{\frac{1}{n}}(s)\right), V^{n, 1 / n}\left(\eta_{\frac{1}{n}}(s)\right)\right)\left(s-\eta_{\frac{1}{n}}(s)\right) \\
& +\alpha\left(X_{i}^{n, 1 / n}\left(\eta_{\frac{1}{n}}(s)\right), V^{n, 1 / n}\left(\eta_{\frac{1}{n}}(s)\right)\right)\left(W(s)-W\left(\eta_{\frac{1}{n}}(s)\right)\right)
\end{aligned}
$$

and

$$
\begin{aligned}
A_{i}^{n, 1 / n}(s)= & A_{i}^{n, 1 / n}\left(\eta_{\frac{1}{n}}(s)\right) \exp \left(\gamma\left(X_{i}^{n, 1 / n}\left(\eta_{\frac{1}{n}}(s)\right), V^{n, 1 / n}\left(\eta_{\frac{1}{n}}(s)\right)\right)\left(B_{i}(s)-B_{i}\left(\eta_{\frac{1}{n}}(s)\right)\right)\right. \\
& +D\left(X_{i}^{n, 1 / n}\left(\eta_{\frac{1}{n}}(s)\right), V^{n, 1 / n}\left(\eta_{\frac{1}{n}}(s)\right)\right)\left(s-\eta_{\frac{1}{n}}(s)\right) \\
& \left.+\beta\left(X_{i}^{n, 1 / n}\left(\eta_{\frac{1}{n}}(s)\right), V^{n, 1 / n}\left(\eta_{\frac{1}{n}}(s)\right)\right)\left(W(s)-W\left(\eta_{\frac{1}{n}}(s)\right)\right)\right)
\end{aligned}
$$

where $D=d-\frac{1}{2}\left(\gamma^{2}+\beta^{2}\right)$. (5.5)-(5.7) then justify the replacement of $A_{i}^{n, 1 / n}(s)$ and $X_{i}^{n, 1 / n}(s)$ by $A_{i}^{n, 1 / n}\left(\eta_{\frac{1}{n}}(s)\right)$ and $X_{i}^{n, 1 / n}\left(\eta_{\frac{1}{n}}(s)\right)$ in the calculations below, where the notation $\approx$ means that the difference converges to zero in probability.

LEMma 5.3. Let $\psi: \mathbb{R}^{2} \rightarrow \mathbb{R}$ be bounded and continuous and have bounded, continuous first derivative $\partial_{2} \psi$ with respect to the second variable. Then

$$
\begin{aligned}
& \int_{0}^{t} \frac{1}{\sqrt{n}} \sum_{i=1}^{n} A_{i}^{n, 1 / n}\left(\eta_{\frac{1}{n}}(s)\right)\left\langle\psi\left(X_{i}^{n, 1 / n}\left(\eta_{\frac{1}{n}}(s)\right), \cdot\right), V^{n, 1 / n}(s)-V^{n, 1 / n}\left(\eta_{\frac{1}{n}}(s)\right)\right\rangle d W(s) \\
\approx & \int_{0}^{t}\left\langle\alpha(\cdot, V(s)) \partial_{2} \psi(*, \cdot)+\beta(\cdot, V(s)) \psi(*, \cdot), V(s) \otimes V(s)\right\rangle d \widetilde{W}^{n}(s) .
\end{aligned}
$$

Proof: Note that

$$
\begin{aligned}
& \int_{0}^{t} \frac{1}{\sqrt{n}} \sum_{i=1}^{n} A_{i}^{n, 1 / n}\left(\eta_{\frac{1}{n}}(s)\right)\left\langle\psi\left(X_{i}^{n, 1 / n}\left(\eta_{\frac{1}{n}}(s)\right), \cdot\right), V^{n, 1 / n}(s)-V^{n, 1 / n}\left(\eta_{\frac{1}{n}}(s)\right)\right\rangle d W(s) \\
= & \int_{0}^{t} \frac{1}{\sqrt{n}} \sum_{i=1}^{n} A_{i}^{n, 1 / n}\left(\eta_{\frac{1}{n}}(s)\right) \frac{1}{n} \sum_{j=1}^{n} A_{j}^{n, 1 / n}(s) \\
& \left(\psi\left(X_{i}^{n, 1 / n}\left(\eta_{\frac{1}{n}}(s)\right), X_{j}^{n, 1 / n}(s)\right)-\psi\left(X_{i}^{n, 1 / n}\left(\eta_{\frac{1}{n}}(s)\right), X_{j}^{n, 1 / n}\left(\eta_{\frac{1}{n}}(s)\right)\right) d W(s)\right. \\
+ & \int_{0}^{t} \frac{1}{\sqrt{n}} \sum_{i=1}^{n} A_{i}^{n, 1 / n}\left(\eta_{\frac{1}{n}}(s)\right) \frac{1}{n} \sum_{j=1}^{n}\left(A_{j}^{n, 1 / n}(s)-A_{j}^{n, 1 / n}\left(\eta_{\frac{1}{n}}(s)\right)\right)
\end{aligned}
$$




$$
\begin{gathered}
\psi\left(X_{i}^{n, 1 / n}\left(\eta_{\frac{1}{n}}(s)\right), X_{j}^{n, 1 / n}\left(\eta_{\frac{1}{n}}(s)\right) d W(s)\right. \\
\approx \int_{0}^{t} \frac{1}{n} \sum_{i=1}^{n} A_{i}^{n, 1 / n}\left(\eta_{\frac{1}{n}}(s)\right) \frac{1}{n} \sum_{j=1}^{n} A_{j}^{n, 1 / n}(s) \partial_{2} \psi\left(X_{i}^{n, 1 / n}\left(\eta_{\frac{1}{n}}(s)\right), X_{j}^{n, 1 / n}\left(\eta_{\frac{1}{n}}(s)\right)\right. \\
\alpha\left(X_{j}^{n, 1 / n}\left(\eta_{\frac{1}{n}}(s)\right), V^{n, 1 / n}\left(\eta_{\frac{1}{n}}(s)\right)\right) d \widetilde{W}^{n}(s) \\
+\int_{0}^{t} \frac{1}{n} \sum_{i=1}^{n} A_{i}^{n, 1 / n}\left(\eta_{\frac{1}{n}}(s)\right) \frac{1}{n} \sum_{j=1}^{n} A_{i}^{n, 1 / n}\left(\eta_{\frac{1}{n}}(s)\right) \beta\left(X_{j}^{n, 1 / n}\left(\eta_{\frac{1}{n}}(s)\right), V^{n, 1 / n}\left(\eta_{\frac{1}{n}}(s)\right)\right) \\
\psi\left(X_{i}^{n, 1 / n}\left(\eta_{\frac{1}{n}}(s)\right), X_{j}^{n, 1 / n}\left(\eta_{\frac{1}{n}}(s)\right) d \widetilde{W}^{n}(s) .\right.
\end{gathered}
$$

The conclusion of the lemma then follows.

ThEOREM 5.2. For $\phi \in \Phi_{\kappa}$, define

$$
\begin{aligned}
& \widetilde{M}_{\phi}(t) \equiv\langle\phi, \widetilde{M}(t)\rangle \\
= & \int_{0}^{t}\left\langle\left(\alpha \partial_{1} \beta\right)(\cdot, V(s)) \phi, V(s)\right\rangle d \widetilde{W}(s) \\
& \left.+\int_{0}^{t}\left\langle\alpha(\cdot, V(s)) \partial_{2} \partial_{3} \beta(*, V(s), \cdot)+\beta(\cdot, V(s)) \partial_{2} \beta(*, V(s), \cdot)\right) \phi(*), V(s) \otimes V(s)\right\rangle d \widetilde{W}(s) \\
& +\int_{0}^{t}\left\langle\left(\alpha \partial_{1} \alpha\right)(\cdot, V(s)) \phi, V(s)\right\rangle d \widetilde{W}(s) \\
& \left.+\int_{0}^{t}\left\langle\alpha(\cdot, V(s)) \partial_{2} \partial_{3} \alpha(*, V(s), \cdot)+\beta(\cdot, V(s)) \partial_{2} \alpha(*, V(s), \cdot)\right) \phi^{\prime}(*), V(s) \otimes V(s)\right\rangle d \widetilde{W}(s),
\end{aligned}
$$

where $\partial_{1}$ and $\partial_{3}$ are derivatives with respect to the corresponding variables and $\partial_{2}$ refers to the operator defined in (S5). Then $\widetilde{M}_{\phi}$ is a martingale satisfying $\left[W, \widetilde{M}_{\phi}\right]_{t}=0$. Let $\widetilde{S}$ be a limit point of $\left\{\widetilde{S}_{n}\right\}$. Then $\widetilde{S}$ is the unique solution of

$$
\langle\phi, \widetilde{S}(t)\rangle=\langle\phi, \widetilde{M}(t)\rangle+\int_{0}^{t}\left\langle F_{1}(V(s)) \phi, \widetilde{S}(s)\right\rangle d s+\int_{0}^{t}\left\langle F_{2}(V(s)) \phi, \widetilde{S}(s)\right\rangle d W(s) .
$$

Proof: Recall that $I_{1}, I_{2}, I_{3}$ are defined by (5.4). It is easy to see that $I_{1} \rightarrow 0$. Note that

$$
\begin{aligned}
& I_{2}= \frac{1}{\sqrt{n}} \sum_{i=1}^{n} \int_{0}^{t} A_{i}^{n, 1 / n}(s) \phi\left(X_{i}^{n, 1 / n}(s)\right) \\
& {\left[d\left(X_{i}^{n, 1 / n}\left(\eta_{\frac{1}{n}}(s)\right), V^{n, 1 / n}\left(\eta_{\frac{1}{n}}(s)\right)\right)-d\left(X_{i}^{n, 1 / n}(s), V^{n, 1 / n}(s)\right)\right] d s } \\
&+ \frac{1}{\sqrt{n}} \sum_{i=1}^{n} \int_{0}^{t} A_{i}^{n, 1 / n}(s)\left[L\left(V^{n, 1 / n}\left(\eta_{\frac{1}{n}}(s)\right)\right) \phi\left(X_{i}^{n, 1 / n}\left(\eta_{\frac{1}{n}}(s)\right)\right)\right. \\
&\left.-L\left(V^{n, 1 / n}(s)\right) \phi\left(X_{i}^{n, 1 / n}(s)\right)\right] d s
\end{aligned}
$$




$$
\begin{aligned}
+\sqrt{n} \int_{0}^{t}\left(\left\langled\left(\cdot, V^{n, 1 / n}(s)\right) \phi\right.\right. & +L\left(V^{n, 1 / n}(s) \phi, V^{n, 1 / n}(s)\right\rangle \\
& -\left\langle d\left(\cdot, V^{n}(s)\right) \phi+L\left(V^{n}(s) \phi, V^{n}(s)\right\rangle\right) d s \\
\equiv I_{21}+I_{22}+I_{23}, &
\end{aligned}
$$

By Lemma 5.1, it is easy to show that $I_{21} \rightarrow 0$ and $I_{22} \rightarrow 0$, and by the definition of $F_{1}$ in $(4.1)$,

$$
I_{23}=\int_{0}^{t}\left\langle F_{1}\left(V^{n}(s), V^{n, 1 / n}(s)\right) \phi, \widetilde{S}_{n}(s)\right\rangle d s
$$

which converges to the second term on the right of (5.14).

Similarly

$$
\begin{aligned}
I_{3}= & \int_{0}^{t} \frac{1}{\sqrt{n}} \sum_{i=1}^{n} A_{i}^{n, 1 / n}(s) \phi\left(X_{i}^{n, 1 / n}(s)\right) \\
& {\left[\beta\left(X_{i}^{n, 1 / n}\left(\eta_{\frac{1}{n}}(s)\right), V^{n, 1 / n}\left(\eta_{\frac{1}{n}}(s)\right)\right)-\beta\left(X_{i}^{n}(s), V^{n}(s)\right)\right] d W(s) } \\
& +\int_{0}^{t} \frac{1}{\sqrt{n}} \sum_{i=1}^{n} A_{i}^{n, 1 / n}(s) \phi^{\prime}\left(X_{i}^{n, 1 / n}(s)\right) \\
& {\left[\alpha\left(X_{i}^{n, 1 / n}\left(\eta_{\frac{1}{n}}(s)\right), V^{n, 1 / n}\left(\eta_{\frac{1}{n}}(s)\right)\right)-\alpha\left(X_{i}^{n}(s), V^{n}(s)\right)\right] d W(s) } \\
& +\int_{0}^{t}\left\langle F_{2}\left(V^{n}(s), V^{n, 1 / n}(s) \phi, \widetilde{S}_{n}(s)\right\rangle d W(s)\right. \\
\equiv & I_{31}+I_{32}+I_{33} .
\end{aligned}
$$

By (5.13) and Lemma 5.3, we have

$$
\begin{aligned}
I_{31} \approx & \int_{0}^{t} \frac{1}{\sqrt{n}} \sum_{i=1}^{n} A_{i}^{n, 1 / n}(s) \phi\left(X _ { i } ^ { n , 1 / n } ( s ) \left(\partial_{1} \beta\left(X_{i}^{n, 1 / n}(s), V^{n, 1 / n}(s)\right)\left(X_{i}^{n, 1 / n}\left(\eta_{\frac{1}{n}}(s)\right)-X_{i}^{n, 1 / n}(s)\right)\right.\right. \\
& \left.\left.\quad+\left\langle\partial_{2} \beta\left(X_{i}^{n, 1 / n}(s), V^{n, 1 / n}(s), \cdot\right), V^{n, 1 / n}\left(\eta_{\frac{1}{n}}(s)\right)\right)-V^{n, 1 / n}(s)\right\rangle\right) d W(s) \\
\approx & \int_{0}^{t}\left\langle\left(\alpha \partial_{1} \beta\right)(\cdot, V(s)) \phi, V(s)\right\rangle d \widetilde{W}^{n}(s) \\
& \left.+\int_{0}^{t}\left\langle\alpha(\cdot, V(s)) \partial_{2} \partial_{3} \beta(*, V(s), \cdot)+\beta(\cdot, V(s)) \partial_{2} \beta(*, V(s), \cdot)\right) \phi(*), V(s) \otimes V(s)\right\rangle d \widetilde{W}^{n}(s)
\end{aligned}
$$

and

$$
\begin{aligned}
I_{32} \approx & \int_{0}^{t}\left\langle\left(\alpha \partial_{1} \alpha\right)(\cdot, V(s)) \phi, V(s)\right\rangle d \widetilde{W}^{n}(s) \\
& +\int_{0}^{t}\left\langle\alpha(\cdot, V(s)) \partial_{2} \partial_{3} \alpha(*, V(s), \cdot)\right. \\
& \left.\left.\quad+\beta(\cdot, V(s)) \partial_{2} \alpha(*, V(s), \cdot)\right) \phi^{\prime}(*), V(s) \otimes V(s)\right\rangle d \widetilde{W}^{n}(s) .
\end{aligned}
$$

Then $I_{31}+I_{32}$ converges to $\langle\phi, \widetilde{M}\rangle$ and $I_{33}$ converges to the third term on the right of (5.14). 
Uniqueness of the solution follows from Lemma 4.2 giving the desired result.

Finally, we combine the results of Sections 4 and 5 .

TheOREM 5.3. Let $S_{n}^{*}(t)=\sqrt{n}\left(V^{n, 1 / n}(t)-V(t)\right)$. Then $S_{n}^{*} \Rightarrow S^{*}$ where $S^{*}$ is the unique solution of the stochastic evolution equation

$$
\begin{array}{r}
\left\langle\phi, S^{*}(t)\right\rangle=\langle\phi, S(0)\rangle+\langle\phi, M(t)+\widetilde{M}(t)\rangle+\int_{0}^{t}\left\langle F_{1}(V(s)) \phi, S^{*}(s)\right\rangle d s \\
+\int_{0}^{t}\left\langle F_{2}\left(V(s) \phi, S^{*}(s)\right\rangle d W(s),\right.
\end{array}
$$

$\phi \in \Phi_{\kappa}$.

REMARK 5.4. Comparing (5.15) to (1.10), we see that the difference lies in the additional martingale driving term $\widetilde{M} . \widetilde{M}$ arises directly from the discrete-time approximation of the driving Brownian motion $W$ through the limit in Lemma 5.2.

\section{Appendix A. Proof of monotonicity.}

We can represent $\Phi_{-q}$ as the space of equivalence classes of $(q+1)$-tuples $v=$ $\left\{\left(v_{0}, v_{1}, \cdots, v_{q}\right)\right\}$, where $v_{j} \in L_{q} \equiv L^{2}\left(\mathbb{R},\left(1+x^{2}\right)^{2 q} d x\right)$, such that

$$
\langle v, f\rangle=\sum_{k=0}^{q} \int_{\mathbb{R}}\left(1+x^{2}\right)^{2 q} v_{k}(x) \frac{d^{k}}{d x^{k}}(f(x) \psi(x)) d x .
$$

The $(q+1)$-tuples $u$ and $v$ are equivalent if the right side of (A.1) does not change when $v$ is replaced by $u$. Then

$$
\|v\|_{-q}^{2}=\inf \left\{\sum_{k=0}^{q}\left\|u_{k}\right\|_{L_{q}}^{2}: u=\left(u_{0}, \ldots, u_{q}\right) \sim v\right\} .
$$

By the Riesz representation theorem, for each $v \in \Phi_{-q}$, there exists a unique $\phi \equiv \theta_{q} v \in$ $\Phi_{q}$ such that

$$
\langle v, f\rangle=\langle\phi, f\rangle_{q}=\sum_{k=0}^{q}\left\langle(\phi \psi)^{(k)},(f \psi)^{(k)}\right\rangle_{L_{q}} .
$$

It follows that

$$
v \sim\left\{\left(\phi \psi,(\phi \psi)^{\prime}, \cdots,(\phi \psi)^{(q)}\right)\right\}
$$

and

$$
\|v\|_{-q}^{2}=\sum_{k=0}^{q}\left\|(\phi \psi)^{(k)}\right\|_{L_{q}}^{2} .
$$

In particular, the infimum in (A.2) is achieved. For each $u=\left\{\left(u_{0}, u_{1}, \cdots, u_{q}\right)\right\} \in \Phi_{-q}$,

$$
\left.\langle u, v\rangle_{-q}=\sum_{k=0}^{q}\left\langle u_{k},(\phi \psi)^{(k)}\right)\right\rangle_{L_{q}}
$$

does not depend on the choice of the $(q+1)$-tuple in the class of $u$. Note also, that since $\Phi_{q+2}$ is dense in $\Phi_{q},\left\{v \in \Phi_{-q}: \theta_{q} v \in \Phi_{q+2}\right\}$ is dense in $\Phi_{-q}$. 
Lemma A.1. Suppose that $f$ and its derivatives up to order $q$ are bounded. Then for $\gamma, \phi \in \Phi_{q+1}$

$$
\begin{aligned}
& \sum_{k=0}^{q} \int_{\mathbb{R}}\left(1+x^{2}\right)^{2 q}\left(f \gamma^{\prime} \psi\right)^{(k)}(\phi \psi)^{(k)} d x \\
= & \int_{\mathbb{R}}\left(1+x^{2}\right)^{2 q} f(\gamma \psi)^{(q+1)}(\phi \psi)^{(q)} d x+O\left(\|\gamma\|_{q}\|\phi\|_{q}\right) \\
= & -\int_{\mathbb{R}}\left(1+x^{2}\right)^{2 q} f(\gamma \psi)^{(q)}(\phi \psi)^{(q+1)} d x+O\left(\|\gamma\|_{q}\|\phi\|_{q}\right),
\end{aligned}
$$

and hence for $\gamma=\phi$,

$$
\sum_{k=0}^{q} \int_{\mathbb{R}}\left(1+x^{2}\right)^{2 q}\left(f \phi^{\prime} \psi\right)^{(k)}(\phi \psi)^{(k)} d x=O\left(\|\phi\|_{q}^{2}\right) .
$$

In addition

$$
\begin{aligned}
& \sup _{\gamma \in \Phi_{q}} \frac{\sum_{k=0}^{q} \int_{\mathbb{R}}\left(1+x^{2}\right)^{2 q}\left(f \gamma^{\prime} \psi\right)^{(k)}(\phi \psi)^{(k)} d x}{\|\gamma\|_{q}} \\
& \quad \leq \sqrt{\int_{\mathbb{R}}\left(1+x^{2}\right)^{2 q}\left|f(\phi \psi)^{(q+1)}\right|^{2} d x}+c_{19} \sum_{1 \leq i, j \leq q} \sqrt{\int_{\mathbb{R}}\left(1+x^{2}\right)^{2 q}\left|f^{(i)}(\phi \psi)^{(j)}\right|^{2} d x}
\end{aligned}
$$

Proof: Let $f_{1}=\frac{f \psi^{\prime}}{\psi}$ and note that if $f$ and its derivatives are bounded, then $f_{1}$ and its derivatives are bounded. Then

$$
\begin{aligned}
& \sum_{k=0}^{q} \int_{\mathbb{R}}\left(1+x^{2}\right)^{2 q}\left(f \gamma^{\prime} \psi\right)^{(k)}(\phi \psi)^{(k)} d x \\
= & \sum_{k=0}^{q} \int_{\mathbb{R}}\left(1+x^{2}\right)^{2 q}\left(f(\gamma \psi)^{\prime}-f_{1} \gamma \psi\right)^{(k)}(\phi \psi)^{(k)} d x \\
= & \sum_{k=0}^{q} \int_{\mathbb{R}}\left(1+x^{2}\right)^{2 q}\left(f(\gamma \psi)^{\prime}\right)^{(k)}(\phi \psi)^{(k)} d x-\sum_{k=0}^{q} \int_{\mathbb{R}}\left(1+x^{2}\right)^{2 q}\left(f_{1} \gamma \psi\right)^{(k)}(\phi \psi)^{(k)} d x \\
= & \int_{\mathbb{R}}\left(1+x^{2}\right)^{2 q} f(\gamma \psi)^{(q+1)}(\phi \psi)^{(q)} d x+\sum_{l=0}^{q-1}\left(\begin{array}{l}
q \\
l
\end{array}\right) \int_{\mathbb{R}}\left(1+x^{2}\right)^{2 q} f^{(q-l)}(\gamma \psi)^{(l+1)}(\phi \psi)^{(q)} d x \\
& \quad+\sum_{k=0}^{q-1} \int_{\mathbb{R}}\left(1+x^{2}\right)^{2 q}\left(f(\gamma \psi)^{\prime}\right)^{(k)}(\phi \psi)^{(k)} d x \\
& \quad-\sum_{k=0}^{q} \int_{\mathbb{R}}\left(1+x^{2}\right)^{2 q}\left(f_{1} \gamma \psi\right)^{(k)}(\phi \psi)^{(k)} d x \\
= & \int_{\mathbb{R}}\left(1+x^{2}\right)^{2 q} f(\gamma \psi)^{(q+1)}(\phi \psi)^{(q)} d x+O\left(\|\gamma\|_{q}\|\phi\|_{q}\right) .
\end{aligned}
$$


Integrating the first term in the fourth expression above by parts gives

$$
\begin{aligned}
-\int_{\mathbb{R}}(1+ & \left.x^{2}\right)^{2 q} f(\gamma \psi)^{(q)}\left((\phi \psi)^{(q+1)}+\frac{4 q x}{1+x^{2}}(\phi \psi)^{(q)}\right) d x \\
& -\int_{\mathbb{R}}\left(1+x^{2}\right)^{2 q} f^{\prime}(\gamma \psi)^{(q)}(\phi \psi)^{(q)} d x \\
& +\sum_{l=0}^{q-1}\left(\begin{array}{l}
q \\
l
\end{array}\right) \int_{\mathbb{R}}\left(1+x^{2}\right)^{2 q} f^{(q-l)}(\gamma \psi)^{(l+1)}(\phi \psi)^{(q)} d x \\
& +\sum_{k=0}^{q-1} \int_{\mathbb{R}}\left(1+x^{2}\right)^{2 q}\left(f(\gamma \psi)^{\prime}\right)^{(k)}(\phi \psi)^{(k)} d x \\
& -\sum_{k=0}^{q} \int_{\mathbb{R}}\left(1+x^{2}\right)^{2 q}\left(f_{1} \gamma \psi\right)^{(k)}(\phi \psi)^{(k)} d x \\
=- & \int_{\mathbb{R}}\left(1+x^{2}\right)^{2 q} f(\gamma \psi)^{(q)}(\phi \psi)^{(q+1)} d x+O\left(\|\gamma\|_{q}\|\phi\|_{q}\right) .
\end{aligned}
$$

Since $\|\gamma\|_{q} \geq\left\|(\gamma \psi)^{(j)}\right\|_{L_{q}}$, (A.3) follows by the Cauchy-Schwartz inequality.

Finally, if $\gamma=\phi$, we can add the two identities to obtain

$$
\sum_{k=0}^{q} \int_{\mathbb{R}}\left(1+x^{2}\right)^{2 q}\left(f \phi^{\prime} \psi\right)^{(k)}(\phi \psi)^{(k)} d x=O\left(\|\phi\|_{q}^{2}\right)
$$

Write $F_{1}=F_{11}+F_{12}$ and $F_{2}=F_{21}+F_{22}$, where

$$
F_{11} \phi=\frac{1}{2} a \phi^{\prime \prime}+b \phi^{\prime}, \quad F_{21} \phi=\alpha \phi^{\prime}
$$

For $\nu \in \mathcal{M}(\mathbb{R})$, let $\rho_{\nu}=\int_{\mathbb{R}} \psi(x)^{-1} \nu(d x)$.

Lemma A.2. For $v \in \Phi_{-q}$ such that $\phi=\theta_{q} v \in \Phi_{q+2}$,

$$
2\left\langle v, F_{11}^{*} v\right\rangle_{-q}=-\int_{\mathbb{R}} a(x, \nu)\left(1+x^{2}\right)^{2 q}\left|(\phi \psi)^{(q+1)}(x)\right|^{2} d x+O\left(\|v\|_{-q}^{2}\right),
$$

where $\left|O\left(\|v\|_{-q}^{2}\right)\right| \leq c_{20}\|v\|_{-q}^{2}$ with $c_{20}$ independent of $\nu$.

Proof: By definition,

$$
\begin{aligned}
\left\langle v, F_{11}^{*} v\right\rangle_{-q} & =\left\langle\phi, F_{11}^{*} v\right\rangle=\left\langle F_{11} \phi, v\right\rangle=\left\langle F_{11} \phi, \phi\right\rangle_{q} \\
=\sum_{k=0}^{q} \int_{\mathbb{R}}\left(1+x^{2}\right)^{2 q}(\phi \psi)^{(k)}\left(\frac{1}{2} a \phi^{\prime \prime} \psi\right)^{(k)} d x & \\
& +\sum_{k=0}^{q} \int_{\mathbb{R}}\left(1+x^{2}\right)^{2 q}(\phi \psi)^{(k)}\left(b \phi^{\prime} \psi\right)^{(k)} d x,
\end{aligned}
$$

where the third equality follows from the fact that $F_{11} \phi \in \Phi_{q}$. By Lemma A.1, the term involving $b$ is bounded by a constant times $\|\phi\|_{q}^{2} \equiv\|v\|_{-q}^{2}$. 
For the term involving $a$, let $a_{1}=\frac{a \psi^{\prime \prime}}{\psi}$ and $a_{2}=\frac{2 a \psi^{\prime}}{\psi}$. Then

$$
\begin{aligned}
& \sum_{k=0}^{q} \int_{\mathbb{R}}\left(1+x^{2}\right)^{2 q}(\phi \psi)^{(k)}\left(a \phi^{\prime \prime} \psi\right)^{(k)} d x \\
= & \sum_{k=0}^{q} \int_{\mathbb{R}}\left(1+x^{2}\right)^{2 q}(\phi \psi)^{(k)}\left(a(\phi \psi)^{(2)}-a_{1}(\psi \phi)-a_{2}\left(\psi \phi^{\prime}\right)\right)^{(k)} d x \\
= & -\sum_{k=0}^{q} \int_{\mathbb{R}}\left(1+x^{2}\right)^{2 q}(\phi \psi)^{(k+1)} a(\phi \psi)^{(k+1)} d x+O\left(\|\phi\|_{q}^{2}\right) \\
= & -\int_{\mathbb{R}} a\left(1+x^{2}\right)^{2 q}\left|(\phi \psi)^{(q+1)}\right|^{2} d x+O\left(\|\phi\|_{q}^{2}\right),
\end{aligned}
$$

where the second equality follows by integrating by parts and applying Lemma A.1.

Lemma A.3. There exists a constant $c_{21}$ such that for $\epsilon>0, v \in \Phi_{-\kappa}$ such that $\phi=$ $\theta_{q} v \in \Phi_{q+2}$, and $\nu \in \mathcal{M}(\mathbb{R})$,

$$
\begin{aligned}
& \int_{U}\left\|F_{21}^{*}(\nu, u) v\right\|_{-q}^{2} \mu(d u) \\
& \quad \leq(1+\epsilon) \int_{\mathbb{R}}\left(1+x^{2}\right)^{2 q} \int_{U}|\alpha(\cdot, \nu, u)|^{2} \mu(d u)\left|(\phi \psi)^{(q+1)}\right|^{2} d x+c_{21}\left(1+\epsilon^{-1}\right)\|v\|_{-q}^{2} .
\end{aligned}
$$

Proof: Noting that

$$
\left\|F_{21}^{*}(\nu, u) v\right\|_{-q}=\sup _{\gamma \in \Phi_{q}} \frac{\left\langle F_{21}^{*} v, \gamma\right\rangle}{\|\gamma\|_{q}}=\sup _{\gamma \in \Phi_{q}} \frac{\left\langle\phi, F_{21} \gamma\right\rangle_{q}}{\|\gamma\|_{q}},
$$

by (A.3),

$$
\begin{aligned}
\left\|F_{21}^{*}(\nu, u) v\right\|_{-q}^{2} \leq\left(\sqrt{\int_{\mathbb{R}}\left(1+x^{2}\right)^{2 q}\left|\alpha(x, \nu, u)(\phi \psi)^{(q+1)}\right|^{2} d x}\right. \\
\left.\quad+c_{19} \sum_{1 \leq i, j \leq q} \sqrt{\int_{\mathbb{R}}\left(1+x^{2}\right)^{2 q}\left|\alpha^{(i)}(x, \nu, u)(\phi \psi)^{(j)}\right|^{2} d x}\right)^{2} .
\end{aligned}
$$

Consequently, there exists a constant $c_{22}$ such that

$$
\begin{aligned}
\left\|F_{21}^{*}(\nu, u) v\right\|_{-q}^{2} \leq(1+\epsilon) & \int_{\mathbb{R}}\left(1+x^{2}\right)^{2 q}\left|\alpha(x, \nu, u)(\phi \psi)^{(q+1)}\right|^{2} d x \\
& +c_{22}\left(1+\epsilon^{-1}\right) \sum_{1 \leq i, j \leq q} \int_{\mathbb{R}}\left(1+x^{2}\right)^{2 q}\left|\alpha^{(i)}(x, \nu, u)(\phi \psi)^{(j)}\right|^{2} d x^{2},
\end{aligned}
$$

and integrating with respect to $\mu$, the boundedness of $\alpha^{(i)}$ in $L^{2}(U, \mu)$ implies the existence of $c_{21}$.

LemMA A.4. For $v \in \Phi_{-\kappa}$,

$$
2\left\langle v, F_{12}^{*} v\right\rangle_{-q} \leq c_{23} \rho_{\nu}\|v\|_{-q}^{2} .
$$


Proof: Assume that $\phi=\theta_{q} v \in \Phi_{q+2}$. As in Lemma A.2,

$$
\left\langle v, F_{12}^{*} v\right\rangle_{-q}=\left\langle F_{12} \phi, \phi\right\rangle_{q} .
$$

Write

$$
\left\langle F_{12}(\nu) \phi, \phi\right\rangle_{q}=\langle d(\cdot, \nu) \phi, \phi\rangle_{q}+\left\langle G_{1}(\nu) \phi, \phi\right\rangle_{q}
$$

where

$$
G_{1}(\nu) \phi=\int_{\mathbb{R}}\left(\partial d(x, \nu, \cdot) \phi(x)+\frac{1}{2} \partial a(x, \nu, \cdot) \phi^{\prime \prime}(x)+\partial b(x, \nu, \cdot) \phi^{\prime}(x)\right) \nu(d x) .
$$

The boundedness of the derivatives of $d$ implies that the first term on the right of (A.6) is $O\left(\|\phi\|_{q}\right)$ uniformly in $\nu$. Note that

$$
|\phi(x) \psi(x)|^{2} \leq \int_{\mathbb{R}}\left|(\phi(y) \psi(y))^{\prime}\right|^{2}\left(1+y^{2}\right)^{2} d y \int_{\mathbb{R}}\left(1+y^{2}\right)^{-2} d y,
$$

so

$$
|\phi(x) \psi(x)| \leq O\left(\|\phi\|_{1}\right) .
$$

Similarly, letting $K_{1}=\sup _{z} \frac{\left|\psi^{\prime}(z)\right|}{\psi(z)}$ and $K_{2}=\sup _{z} \frac{\left|\psi^{\prime \prime}(z)\right|}{\psi(z)}$,

$$
\left|\phi^{\prime}(x) \psi(x)\right| \leq\left|(\phi(x) \psi(x))^{\prime}\right|+K_{1}|\phi(x) \psi(x)| \leq O\left(\|\phi\|_{2}\right)
$$

and

$$
\left|\phi^{\prime \prime}(x) \psi(x)\right| \leq\left|(\phi(x) \psi(x))^{\prime \prime}\right|+2 K_{1}\left|\phi^{\prime}(x) \psi(x)\right|+K_{2}|\phi(x) \psi(x)| \leq O\left(\|\phi\|_{3}\right) .
$$

Consequently,

$$
\begin{aligned}
\left\|G_{1}(\nu) \phi\right\|_{q}= & \int_{\mathbb{R}}\left(\|\partial d(x, \nu, \cdot)\|_{q}|\phi(x) \psi(x)|+\frac{1}{2}\|\partial a(x, \nu, \cdot)\|_{q}\left|\phi^{\prime \prime}(x) \psi(x)\right|\right. \\
& \left.+\|\partial b(x, \nu, \cdot)\|_{q}\left|\phi^{\prime}(x) \psi(x)\right|\right) \psi^{-1}(x) \nu(d x) \\
\leq & c_{31} \rho_{\nu}\|\phi\|_{3} \leq c_{31} \rho_{\nu}\|\phi\|_{q}=c_{31} \rho_{\nu}\|v\|_{-q} .
\end{aligned}
$$

Combining this inequality with the estimate on the first term gives the result.

Lemma A.5. There exists $c_{24}$ such that for $v \in \Phi_{-\kappa}$,

$$
\int_{U}\left\|F_{22}^{*}(\nu, u) v\right\|_{-q}^{2} \mu(d u) \leq c_{24} \rho_{\nu}^{2}\|v\|_{-q}^{2} .
$$

Proof: To bound $\left\|F_{22}^{*} v\right\|_{-q}$, note that

$$
\left\|F_{22}^{*} v\right\|_{-q}=\sup _{\gamma \in \Phi_{q+2}} \frac{\left\langle\gamma, F_{22}^{*} v\right\rangle}{\|\gamma\|_{q}}=\sup _{\gamma \in \Phi_{q+2}} \frac{\left\langle F_{22} \gamma, \phi\right\rangle_{q}}{\|\gamma\|_{q}} \leq \sup _{\gamma \in \Phi_{q+2}} \frac{\left\|F_{22} \gamma\right\|_{q}}{\|\gamma\|_{q}}\|\phi\|_{q} .
$$

Then $\left\|F_{22} \gamma\right\|_{q} \leq c_{25}(u) \rho_{\nu}\|\gamma\|_{q}$ by the same argument used to estimate $G_{1}$ in Lemma A.4, and $c_{25}$ can be selected to be integrable with respect to $\mu$. 
Combining the previous lemmas we have

Lemma A.6. For $v \in \Phi_{-\kappa}$,

$$
2\left\langle v, F_{1}^{*}(\nu) v\right\rangle_{-q}+\int_{U}\left\|F_{2}^{*}(\nu, u) v\right\|_{-q}^{2} \mu(d u) \leq c_{18} \rho_{\nu}^{2}\|v\|_{-q}^{2} .
$$

Proof: Selecting $\epsilon>0$ so that $(1+\epsilon)^{2} \leq(1+\delta)$ for $\delta$ in $\left(S_{4}\right)$,

$$
\begin{aligned}
& 2\left\langle v, F_{1}^{*}(\nu) v\right\rangle_{-q}+\int_{U}\left\|F_{2}^{*}(\nu, u) v\right\|_{-q}^{2} \mu(d u) \\
\leq & 2\left\langle v, F_{11}^{*}(\nu) v\right\rangle_{-q}+(1+\epsilon) \int_{U}\left\|F_{21}^{*}(\nu, u) v\right\|_{-q}^{2} \mu(d u) \\
& +2\left\langle v, F_{12}^{*}(\nu) v\right\rangle_{-q}+\left(1+\epsilon^{-1}\right) \int_{U}\left\|F_{22}^{*}(\nu, u) v\right\|_{-q}^{2} \mu(d u) \\
\leq & -\int_{\mathbb{R}}\left(a(x, \nu)-(1+\epsilon)^{2} \int_{U}|\alpha(x, \nu, u)|^{2} \mu(d u)\right)\left(1+x^{2}\right)^{2 q}\left|(\phi \psi)^{(q+1)}(x)\right|^{2} d x \\
& +\left(c_{20}+c_{21}(1+\epsilon)\left(1+\epsilon^{-1}\right)+c_{23} \rho_{\nu}+\left(1+\epsilon^{-1}\right) c_{24} \rho_{\nu}^{2}\right)\|v\|_{-q}^{2} \\
\leq & c_{18} \rho_{\nu}^{2}\|v\|_{-q}^{2},
\end{aligned}
$$

since the first term in the third expression is less than or equal to zero by $(\mathrm{S} 4)$.

Acknowledgement: Part of this work was done when the second author visited WIAS and the University of Wisconsin. He would like to thank both institutions for their hospitality.

\section{REFERENCES}

[1] Pierre Bernard, Denis Talay, and Luciano Tubaro, Rate of convergence for the Kolmogorov equation with variable coefficients, Math. Comp. 63, 555-587, 1994.

[2] Patrick Billingsley, Convergence of Probability Measures, Wiley, New York, 1968.

[3] Donald L. Burkholder, Distribution function inequalities for martingales, Ann. Prob. 1, 19-42, 1973.

[4] T. Chiang, G. Kallianpur, and P. Sundar, Propagation of chaos and McKean-Vlasov equation in duals of nuclear spaces, Appl. Math. Optim. 24, 55-83, 1991.

[5] Dan Crisan, Pierre Del Moral, and Terry Lyons, Discrete filtering using branching and interacting particle systems, Markov Process, Related Fields 5, 293-318, 1999.

[6] Dan Crisan, Piere Del Moral, and Terry Lyons, Interacting particle approximations of the Kushner Stratonovich equation, Adv. Appl. Probab. 31, 819-838, 1999.

[7] Dan Crisan, Jessica Gaines, and Terry Lyons, Convergence of a branching particle method to the solution of the Zakai equation, SIAM J. Appl. Math. 58, 1568-1590, 1998.

[8] Dan Crisan and Terry Lyons, A particle approximation to the solution of the KushnerStratonovitch equation, Probab. Theory and Related Fields 115, 549-578, 1999.

[9] Donald A. Dawson and Jean Vaillancourt, Stochastic McKean-Vlasov equations, NoDEA Nonlinear Differential Equations Appl. 2, 199-229, 1995.

[10] Pierre Del Moral, Non-linear filtering using random particles, Theory Probab. Appl. 40, 690$701,1995$.

[11] P. Del Moral, M.A. Kouritzin, and L. Miclo, On a class of discrete generation interacting particle systems, Electron. J. Probab. 6, 2001.

[12] G.B.Di Masi, M. Pratelli, and W.J. Runggaldier, An approximation for the nonlinear filtering problem with error bound, Stochastics 14, 247-271, 1985.

[13] Stewart N. Ethier and Thomas G. Kurtz, Markov Processes: Characterization and Convergence, Wiley, New York, 1986.

[14] Patrick Florchinger, Le Gland, and François, Time-discretization of the Zakai equation for diffusion processes observed in correlated noise, Stochastics Rep. 35, 233-256, 1991. 
[15] Patrick Florchinger, Le Gland, and François, Time-discretization of the Zakai equation for diffusion processes observed in correlated noise, in Analysis and optimization of systems (Antibes, 1990), 228-237, Lecture Notes in Control and Inform. Sci. 144, Springer, Berlin.

[16] Patrick Florchinger, Le Gland, and François, Particle approximations for first order stochastic partial differential equations, Appl. stochastic anal., New Brunswick, NJ, 121-133, 1991, Lecture Notes in Control and Inform. Sci. 177 Springer, Berlin, 1992.

[17] I.M. Gel'fand and N.Ya Vilenkin Generalized Functions, Vol. 4: Applications of Harmonic Analysis, Academic Press, New York - London, 1964.

[18] Carl Graham, Nonlinear Itô-Skorohod equations and martingale problem with discrete jump sets, Stochastic Process Appl. 40, 69-82, 1992.

[19] Masuyuki Hitsuda and Itaru Mitoma, Tightness problem and stochastic evolution equation arising from fluctuation phenomena for interacting diffusions, J. Multivariate Anal. 19 $311-328,1986$.

[20] Y. Hu, Gopinath Kallianpur, and Jie Xiong, An approximation for the Zakai equation, Appl. Math. Optim. 45, 23-44, 2002.

[21] Gopinath Kallianpur and Jie Xiong, Stochastic Differential Equations in Infinite Dimensional Spaces, IMS Lecture Notes-Monograph Series 26, Institute of Mathematical Statistics, 1995.

[22] Gopinath Kallianpur and Jie Xiong, Asymptotic behavior of a system of interacting nuclearspace-valued stochastic differential equations driven by Poisson random measures, Appl. Math. Optim. 30, 175-201, 1994.

[23] Peter Kotelenez, A class of quasilinear stochastic partial differential equation of McKeanVlasov type with mass conservation, Probab. Theory Relat. Fields 102, 159-188, 1995.

[24] Thomas G.Kurtz, Semigroups of conditioned shifts and approximation of Markov processes, Ann. Probab. 3, 618-642, 1975

[25] Thomas G. Kurtz and Philip Protter, Weak convergence of stochastic integrals and differential equations, II. Infinite-dimensional case, Probabilistic models for nonlinear partial differential equations, 197-285, Lecture Notes in Math. 1-627, Springer, New York, 1996.

[26] Thomas G. Kurtz and Jie Xiong, Particle representations for a class of nonlinear SPDEs, Stochastic Process Appl. 83, 103-126, 1999.

[27] Thomas G. Kurtz and Jie Xiong, Numerical solutions for a class of SPDEs with application to filtering, Stochastics in Finite and Infinite Dimension: In Honor of Gopinath Kallianpur, Edited by T. Hida, R. Karandikar, H. Kunita, B. Rajput, S. Watanabe and J. Xiong, Trends in Math. Birkhauser, 233-258, 2000.

[28] Sergey Lototsky and Boris L. Rozovskii, Recursive multiple Wiener integral expansion for nonlinear filtering of diffusion processes, in Stochastic Processes and Functional Analysis, Riverside, CA, 199-208, 1994, Lecture Notes in Pure and Appl. Math. 186, Dekker, New York, 1997.

[29] Sergey Lototsky, R. Mikulevicius, and Boris L. Rozovskii, Nonlinear filtering revisited: a spectral approach, SIAM J. Control Optim. 35, 435-461, 1997.

[30] Henry P. McKean, Propagation of Chaos for a Class of Non-linear Parabolic Equations, Lecture Series in Differential Equations 2, 177-194, 1967.

[31] Sylvie Méléard, Asymptotic behavior of some interacting particle systems, McKean-Vlasov and Boltzmann models, Probabilistic Models for Nonlinear Partial Differential Equations, Lecture Notes in Math. 1627, 42-95, 1996.

[32] Itaru Mitoma, Tightness of probabilities on $C\left([0,1], \mathcal{S}^{\prime}\right)$ and $D\left([0,1], \mathcal{S}^{\prime}\right)$, Ann. Probab. 11, 989-999, 1983.

[33] Itaru Mitoma, An $\infty$-dimensional inhomogeneous Langevin's equation, J. Funct. Anal. 61, 342-359, 1985

[34] P.L. Morien, Propagation of chaos and fluctuations for a system of weakly interacting white noise driven parabolic SPDE's, Stochastics Stochastics Reps. 58, 1-43, 1996.

[35] J. Picard, Approximation of nonlinear filtering problems and order of convergence, Filtering and Control of Random Processes, Lecture Notes Control Inf. Sci. 61, Springer, New York, 1984.

[36] Boris L. Rozovskii, Stochastic Evolution Systems. Linear Theory and Applications to Nonlinear Filtering, Kluwer Academic Publishers Group, Dordrecht, 1990.

[37] John Walsh, An introduction to stochastic partial differential equations, École d'été de probabilités de Saint Flour., Lecture Notes in Math. 1180, 265-439. Springer, New York, 1984. 